University of Windsor

Scholarship at UWindsor

1974

\title{
On a certain boundary value problem and its relevance in the theory of surface induction hardening.
}

Joseph Po-Shing. Chow

University of Windsor

Follow this and additional works at: https://scholar.uwindsor.ca/etd

\section{Recommended Citation}

Chow, Joseph Po-Shing., "On a certain boundary value problem and its relevance in the theory of surface induction hardening." (1974). Electronic Theses and Dissertations. 2913.

https://scholar.uwindsor.ca/etd/2913

This online database contains the full-text of PhD dissertations and Masters' theses of University of Windsor students from 1954 forward. These documents are made available for personal study and research purposes only, in accordance with the Canadian Copyright Act and the Creative Commons license-CC BY-NC-ND (Attribution, Non-Commercial, No Derivative Works). Under this license, works must always be attributed to the copyright holder (original author), cannot be used for any commercial purposes, and may not be altered. Any other use would require the permission of the copyright holder. Students may inquire about withdrawing their dissertation and/or thesis from this database. For additional inquiries, please contact the repository administrator via email (scholarship@uwindsor.ca) or by telephone at 519-253-3000ext. 3208. 

$r$

ON A CERTAIN BOUNDARY VALUE PROBLEM:

AND ITS RELEVANCE IN THE THEORY OF

SURFACE INDUCTIION HARDENING :

\section{by}

$<$

JOSEPH PO-SHING CHOW

y

A Dissertation

Submitted to the Faculty of Graduate Studies through tho Department of Mathematics in Partial Fulfillment of the Requirements for the Degree of Doctor of Philosophy

At the University of Windsor wirdsor ontario

1973 

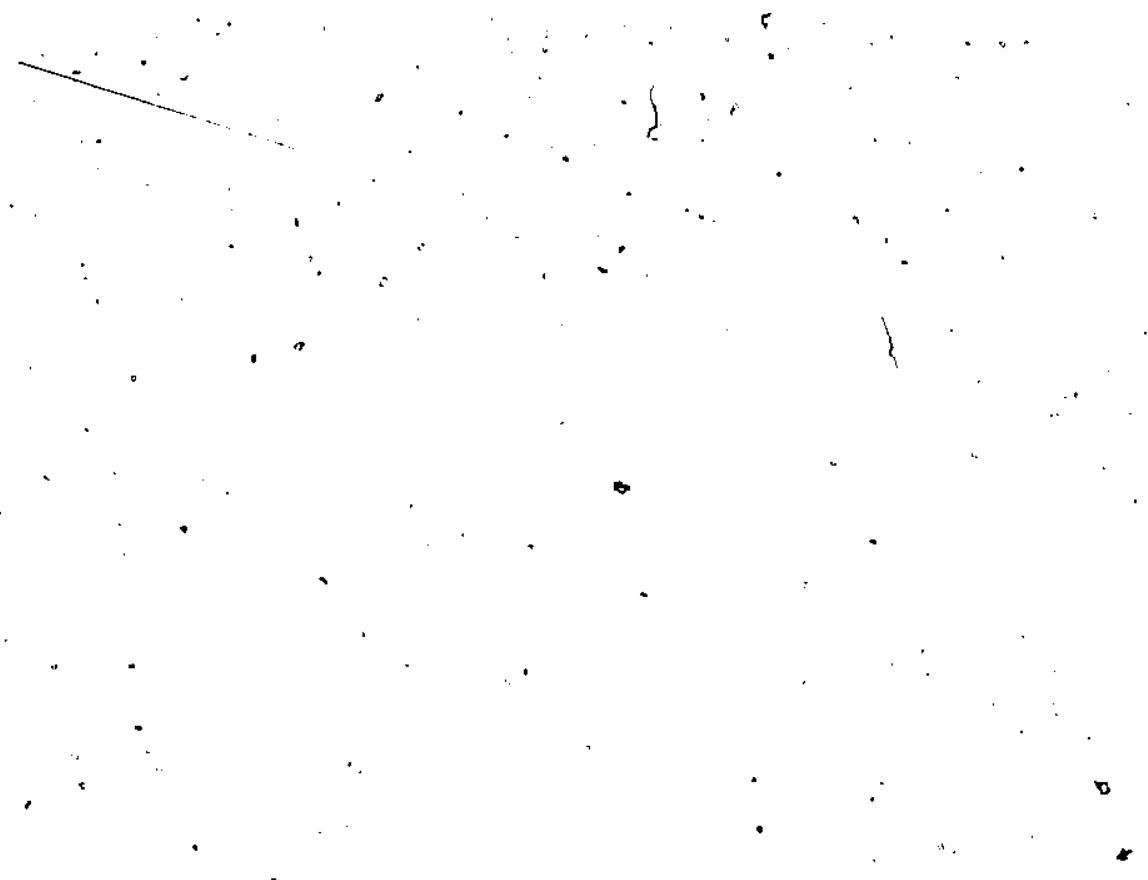

b<smiles>CPC</smiles>

(C) Joseph Pơ-Shing Chow 1974 


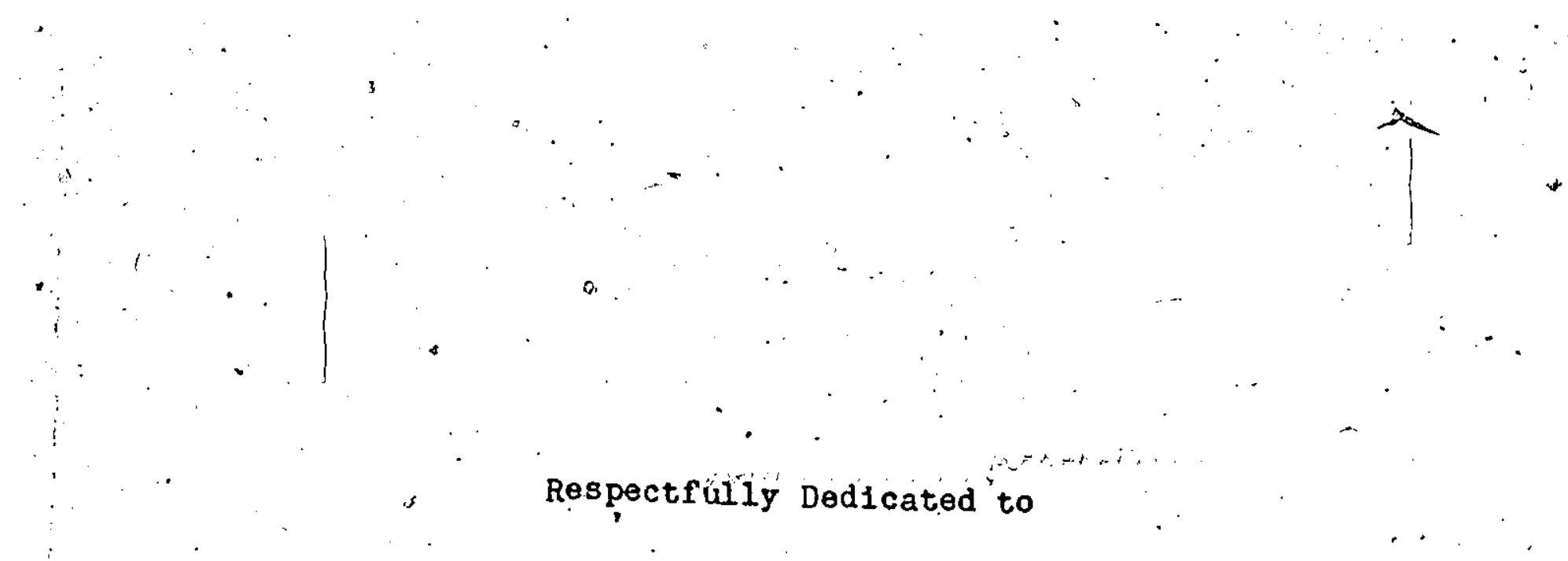

\section{My FATHER and MOTHER}

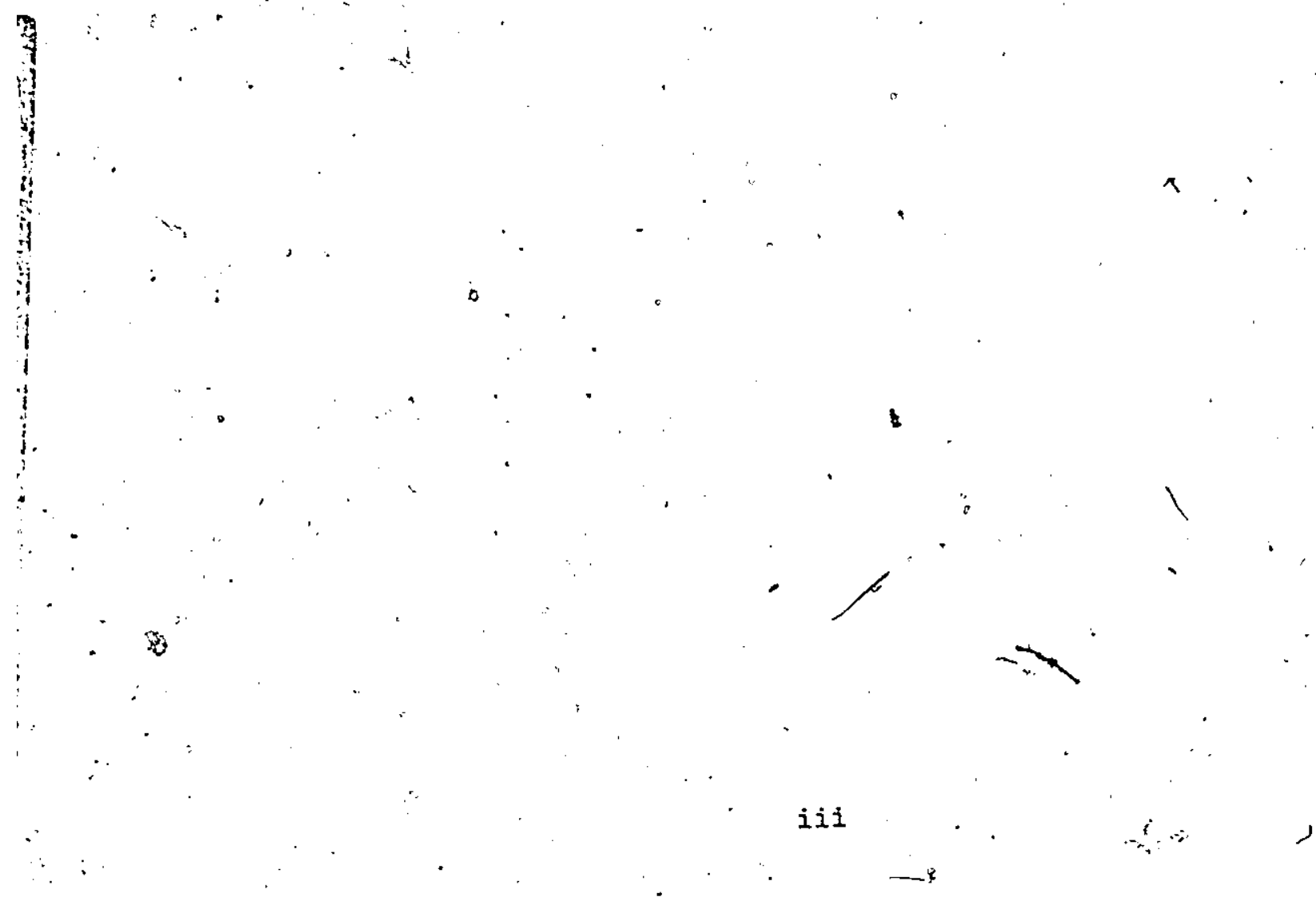




\section{ABSTRACT}

Surface hardening accomplished by localized heating and controlled cooling permits resistance to local surface wear to be combined with good torsional and bending. strength characteristics using cheaper. alloys in mechantcal components. Induction heating ist a convenient means of applying this local heating. Although fast, convenient, and having excellent energy conversion characteristics, it is not simple to set up, nor rapid nor rellable due to the lack of easily useable mathematical solutions to the problem

This study treats the case of induction hardening of a cylindrical metal rod of sufficient length that end effects may be ignored. The boundary vaIue problem is given and a simplified representation developed. The physical parameters, such as conductivity and permeability, are assumed to be smooth functions of the radius of the rod, contrary to earlier studies which assume these to. be constants in order to simplify the mathematics.

A representation theorem for the solution of the problew is proved using a number of lemmas which are stated and proven first. A number of methods for approximating the solution for numerical calculations are also described. Suggestions are made for some possible further studies of the problem. 


\section{ACKNOULEDGEMENTS}

The author wishes to express his sincere appreciation to his supervisor, Dir. K. A. Zischka, for his guidance and encouragement throughout the course of this investigation, for his helpful suggestions and patience during the research and preparation of this dissertation, and for serving as Co-chairman of his woctoral combittee.

To Dr."J. F. MeDonald goes his heartfelt thanks for his aid and guldance guring the author's entire stay at the University of lindsor, and particularly during Dr. Zischkka's absence on sabbatical leave, and for actinf as co-chairman of his Joctoril comittee.

Gratitude is, also extended to the other members of the foctoral Comiltee. These were bean ur. Nickel who served Esternal Examiner, ur. ürake of the Pnysics \$ vepartment, and ur. ifley of the vepartment of tathematics.

Rev. D. T. Faught, Head of the Department of Nathematics, merits special remembrance for his conthulng personal interest in the author's studies which interest included the mundane but welcome provision of graduate assistantships. 
The entire Department of Mathematics; faculty, staff members, and fellow students, have also. contributed much to the success and enjoyment of the author's work and life at Windsor, and he. acknowledges with gratitude this debt.

The work was supported in part by - research - Grants and bursaries from the National Rësearch Counqil of Canada.

- Finally, the author wishes to acknowledge the .. encourasement and support during the whole course of his graduate studies of his parents, to whom this thesis is dedicated.




TABLE of CONTENTS



CHAPTER 3 - THE REPRESENTATION THEOREM

1. 'The Statement of the Representation Theorem

2. Some Fundamental Lemmas

3. The Proof of the, Theorem

CHAPTER 4 - APPROXIMATE METHODS

44

1. The Layer Method

46

2. The Method of Integral Equations

3. The Porturbation Niethod

4. Some Error Estimates

CHAPTER 5 - CONCLUSION

REFERENCES

62

VITA AUCTORIS 


\section{LIST OF FIGURES}

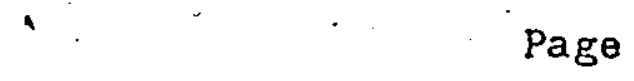

FIGURE I

(') FIGURE 2

8

16

F IGURE 3

$\square$ 


\section{Chapter 1 \\ INTRODUCTION}

\section{(a) Historical Sketch}

The surface hardening of metals and alloys, which are the most widely utilized basic materials for constructing all kinds of machines and mechanisms, plays an important role in modern industilal technology and engineering. Localized heating of individual regions of compoinents is necessary in order to carry out the widely employed industrial process of surface hardening. The possibility of localized heating of surface regions of components of various mechanisms and also the duration of the process depends a great deal upon the heating method selected. Two heating methods are in common use: the application of external heat; and the generation of heat within the work piece.

The application of external heat is the traditional method used and consists of placing the work piece in a hot environment such as a furnace or crucible. This has the disadvantages characterized by a low rate of temperature increase in the work piece (usually of the order of tens or hundreds of degrees per minute), and by the low thermal efficiency of the furnace, both characteristics being unsultable for surface hardening. 
When applying external heat; not more than one-third of the thermal energy is utilized directly in heating the workplece, the remalning heat being-expended In heating the furnace 1tself and in other losses. The result of this is a long heafing process and high speed specific expendíture of thermal energy in heating each kilogram of the material being heated. In addition to this, the localized heating: of the surface regions whioh is required for some articles is practically impossible using a furnace or equivalent means $[10]$.

The method of generating heat within the work plece: usually uses the principle of finduction. It is based on the fact that an eddy current is induced at the surface of an electric conductor which is subjected to a rapidly chànging magnetic field. "This current, flowing as it does through only the very thin surface layer of the conductor, will produce great heat because the Ohmis losses are concentrated there. The magnetic field used in surface hardening a cylindrical steel rod 18,produced by passing a high frequency electric current through a c1rcular coll moving with constant speed with respect to the cylinder. The chief advantages of this form of heating are the speed of the process, the ability to control the degree of hardening by selecting the proper speed of the moving coll, and the ability to harden only a limited portion of the. metal object. Also, objects case hardened by induction 
combline greatly improved torsional and bending fatigue strength with good war resistance while using cheaper alloys. The wide range of present applications to other flelds where speed and convenience of heating, are necessary or desirable, or where local heating is a decided asset, shows the potential for the process if the set up of the process, for, a given application can be made simple, rapid, and reliable.

The next advance in the application of this most useful technique of induction heating is the theoretical study of the process and development of a mathematicat traatment of the process. Until the outcome of $K$. A. Zischka's work [14], not much work had been done to contribute to the mathematical aspect of induction heating. However, in recent years, there has been an increased

- Interest in the construction of mathematical models for Iron and steel work processing $[/ / 3]$ : This has arisen because the posisibility of performing by computer the arduous arithmetic involved in translating a model into numerical results for use in specific cases has now won universal acceptance. The development of a mathematical model depends on a knowledge of the behaviour of the system being studied, the capabilitiy of expressing these mathematically, and on the abllity to solve, the resulting oquations. Unfortunately, the exact mathematical description in closed form of the heating of a work piece 
Is possible only for a few simple shapes of conductors (work pieces) and configurations of the coll. Even with this limitation, the mathematical theory of the induction hardening process is far from complete, mainly because of the great difficulty encountered in solving the a sooclated boundary value problem which usually consists of a system of partial differential equations with coupled boundary conditions. As a result, the physical parameters involved, such as electrical conductivity and permeability, are frequently assumed to be constant even though this is not the true case for induetion hardening [4-6], $[14-15]$ In fact, these physical parameters of permeability and conductivity depend upon the spatial coordinates since they are functions of teruperature, and the temperature depends upon the spatial coordinates because the induction heating process impresses a steep temperature gradient upon the work piece. It is this aspect of the problem. with which we concern ourselves in this thesis.

(b) Scope of the Prosent Work

The present work is intended to treat a boundary value problem arising from the surface induction hardening of a cylindrical metal rod. We shall assume that the physical parameters, such as the electric conductivity. and permeability of this rod, are smooth functions of the radius of the rod: 
In section 1 of chapter 2 , we give a description of the physical model together with its geometry. In section 2 of chapter 2 , we give a brief mathematical formulation of the boundary value problem for this model.

- Some approximations have been made in order that the boundary value problem shall become solvable.

Chapter 3 deals with the solution of this boundary value problem. A representation theorem is proved. In section 1 of chapter 3, the representation theorem is stated whth its pertinent assumptions. In section 2 of chapter 3, some fundamental lemmas which are essential in proving the theorem are stated and proved. In section 3 of chapter 3, we give a proof of the theorem in a form which has been simplified with the help of the lemmas proved in the previous section.

Chapter 4 is chiefly devoted to approximate methods. In section 1 of chapter 4, we use the layer method to approximate the whole system. In section 2 of chapter 4 , we use a method of integral equations to approximate the solution of the second order differential equation which arises in the solution of the boundary value problem. In section 3 of chapter 4, we use a perturbation method to approximate the differential equation for small values of $x$. In section 4 of chapter 4 , we give bounds for replacing the Improper integral by an integral taken over a finite interval. 
$c$

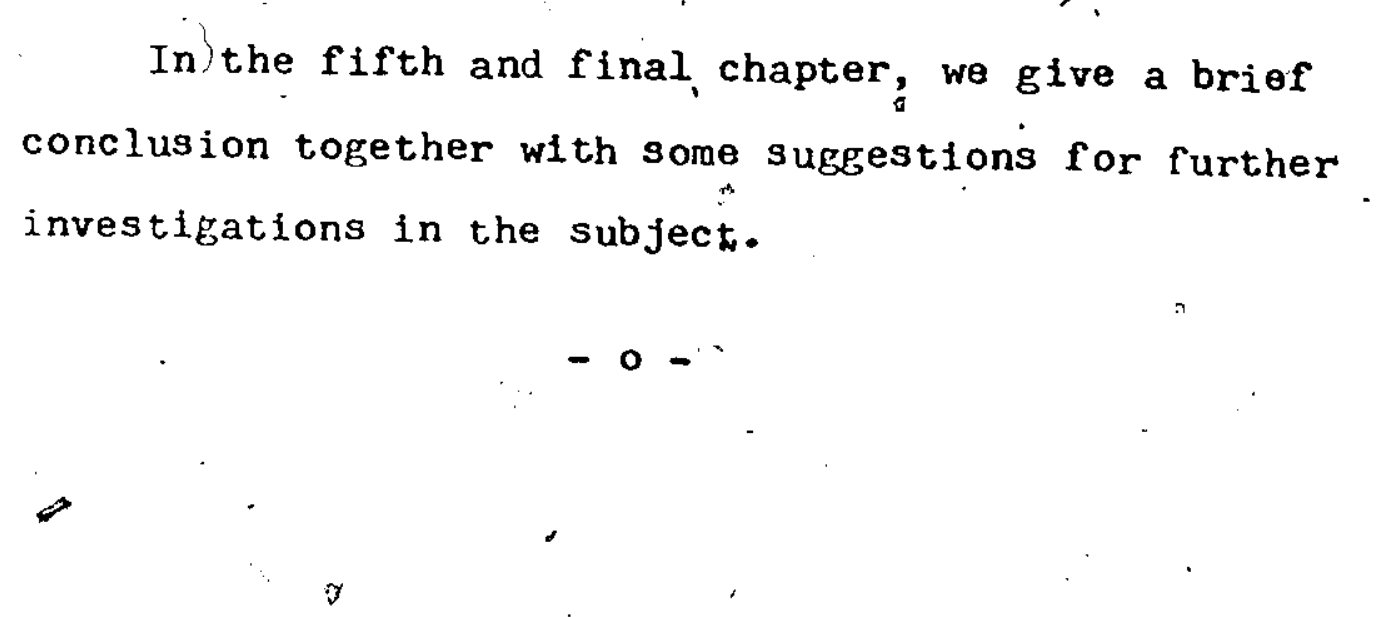

In the fifth and final, chapter, we give a brief 作 $\checkmark$ 
Chapter 2

THE PHYSICAL MODEL AND ITS MATHEMATICAL FORMULATION

This chapterdeals with the physical model of the surface induction hardening of a metallic cylindrical rod. A and the mathematical formulation of the boundary value problem associated with this model. Certain approximations and simplifying assumptions have to be made in order that the problem so described becomes solvable.

1. A Description and the Geometry of the Physical Model

In this thesis we consider the following physical problem. A long cylindrical metallic rod is surrounded by a concentric cylindrical coil of length 2L which moves

- with constant velocity $\vec{v}$ parallel to the axis of the cylinder. The length of the rod is assumed to be much greater than that of the coil so that we can neglect the end effects and treat the rod as having infinite length. Since the velocity $\vec{v}$ of the coil is negligible compared to that of 11ght, we 1gnore the relativistic effect and use only the Newtonian transformation $u=z$ - vi in corder to consider the coordinate system of the coil as a moving frame. The geometry and coordinate system being used are illustrated in Fig. 1. 




$\therefore 0$

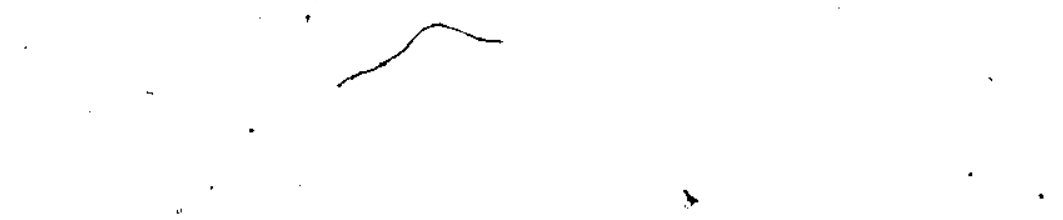

Fig. 1

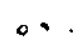


We wish to investigate the effect on the rod when a high frequency (or order $10^{6}$ Hertz) alternating current is passed through the cofl. The current induced in the. work piece by the motion of the coil is neglected as being. insignificant compared to that induced by the alternating magnetic field at the frequencies being used.

2. The Mathematical Formulation of the Boundary Value Problem

The bastc equations governing this problem are $I$ Maxwell's equations. In rationalized MKS units, the macroscopic Maxwell equations may be written as:

(2.1) $\nabla \times \vec{H}=\vec{J}+\frac{\partial \vec{D}}{\partial t}$

(2.2) $\nabla \times \overrightarrow{\mathrm{E}}=-\frac{\partial \overrightarrow{\mathrm{B}}}{\partial t}$

$(2.3)$ Div $\overrightarrow{\vec{D}}=\rho$, and
$(2.4)$ Div $\vec{B}=0$.
Here $\vec{H}$ is the marnetic intensity, $\vec{J}$ is the current density, $\vec{E}$ is the electric fleld, $\vec{D}$ is the displacement vector, $\vec{B}$ is the magnetic induction field, and $\rho$ is the charge density. For the rance of frequencies we are considering, the current becomes the quasistationary case $[4],[14],[15]$, and the displacement current $\partial \vec{D} / \partial t$ is nepligible compared to the true current density $\vec{J}$, and we may replace $(2.1)$ by: 
(2.5) Curl $\vec{H}=\vec{J}$.

Also, from Ohm's Law we have

(2.6) $\vec{J}=\dot{\sigma} \overrightarrow{\mathrm{E}}$,

for nonferromagnetic material we have

(2.7) $\vec{B}=\mu \vec{H}$

while for isotropic material we have

(2.8) $\quad \vec{D}=\varepsilon \vec{E}$; .

where $\sigma$ is the conductivity, $\mu$ the permeability, and

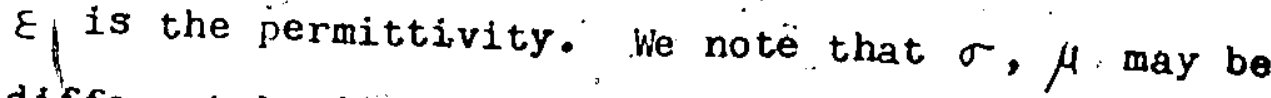
different in different regions. We shall assume the following: Within the $\operatorname{rod} \mu$ and $\sigma$ may depend upon the radial coordinate $r$ because of the high temperature gradient in the radial direction of the rod. Within the coll $\mu$ and $\sigma$ are constant, $\mu=\mu_{c}, \sigma=\sigma_{c}$. In the air $\sigma=0$, and $\mu=\mu_{0}$.

If we assume the electric field $\vec{E}$ and the magnetic intensity $\vec{H}$ to be separable in the time variable, that is $(2.9)(a) \quad \vec{E}=\hat{E} \cdot e^{i \omega t}$, and (2.9) (b) $\quad \vec{H}=\hat{H} e^{1 \omega t}$. where $\widehat{E}$ and $\hat{H}$ are the amplitudes of the electric field $\vec{E}$ and the magnetic Intensity $\vec{H}$ respectively, and $\omega$ is the frequency of the alternating current flowing in the coil, we then have: 
(2.10) $\nabla \cdot x \hat{H}=\dot{\sigma} \hat{E}$, and.

(2.11) $\nabla \times \hat{E}=-1 \omega \hat{B}$.

Since $\hat{B}$ has zero divergence, it may always be represented as the curl of $a$ vector potential, that is (2.12) $\hat{B}=\nabla \times \hat{A}^{\prime}$. .

From the formula we then have curl $(\hat{E}+1 \omega \hat{A})=0$.

It follows that

$(2.13) \quad \hat{E}=-1 \omega \hat{A}$

with $\hat{A}$ defined through. (2.12), is a particular solution of $(2.11)$ : We have within the rod

$(2.14)(a) \quad \nabla \times(\nabla \times \hat{A})=k^{2} \hat{A}+\frac{\operatorname{grad} \mu}{\mu} \times(\nabla \times \hat{A})$

while in the air

$(2.14)(\mathrm{b}) \cdot \nabla \times(\nabla \times \hat{A})=0$,

where

(2.1.5). $k^{2}=-i \omega \mu \sigma$.

In setting up the coil we require that the current density $\hat{J}$ in the coil satisfies the following conditions: $(2.16)(a) \quad \nabla \times \hat{J}=0$, $(2.16)(b) \hat{J}=\left\{0, J_{\vec{r}}, 0\right\}, \quad$ and $(2.16)(c) \quad f_{\varphi} \doteq J_{p}(r) \quad$ for $R_{1} \leq r \leq R_{z}$. That is to say, we consider that the coil consists of solid rings.

From (2.16) (a) - (c), we have the differential equation $\frac{d}{d r}\left(\cdot r j_{\varphi}\right)=0$.

Integrating this differential equation, we obtain $j_{\varphi}=c / r$, where $c$ is the constant of integration. 
- Since the total current I flowing in the coil is

" kept constant, we have

$$
\therefore \quad I=\int_{0}^{2 L} \int_{R}^{R_{2}} j_{6} d r d u=2 L C \ln \left(\frac{R_{2}}{R_{i}}\right) \text {. }
$$

and hence

$$
\text { (2.17) } j_{\varphi}(r)=\frac{I}{2 L \ln \left(R_{2} / R_{1}\right)} \frac{1}{r} \text { for } R_{1} \leq r \leq R_{2} \text {. }
$$

Furthermore, we have the following boundary conditions at the interface of the cylindrical rod and the surroundimg alr:

$$
\begin{aligned}
(2.18)(a) & \hat{E}_{t}^{(r)}=\hat{E}_{t}^{(a)}, \\
\text { (b) } \hat{E}_{n}^{(r)} & =\hat{E}_{t}^{(a)}, \text { and } \\
\text { (c) } \mathrm{B}_{t}^{(r)} & =\frac{\mu}{\mu_{0}} \mathrm{~B}_{t}^{(4)},
\end{aligned}
$$

where the index $(r)$ denotes the interior of the rod and the index (a) denotes the exterior region (air). The subscripts $t$ and $n$ sienify the tangential component and the normal component respectively.

We also have the following boundary conditions at the interface of the coil and the surrounding air

$$
\begin{aligned}
(2.19) \text { (a) } \hat{E}_{t}^{(c)} & =\hat{E}_{t}^{(a)}, \\
\text { (b) } \hat{E}_{n}^{(c)} & =\hat{E}_{n}^{(a)}, \text { and } \\
\text { (c) } \frac{1}{\mu_{c}} B_{t}^{(k)} & -\frac{1}{\mu_{0}} B_{t}^{(a)}=j_{s \varphi},
\end{aligned}
$$


where the index (c) denotes the interior of the coil and $f_{s p}$ is the surface current density at the interface.

We obviously must require that $\hat{E}$ and $\hat{B}$ remain bounded along the axis of the rod, and that $\hat{E}$ and $\hat{B}$ are functions of order $\left(? / r^{2}\right)$ for large values of $r$, that is, $\hat{E}$, and $\hat{B}$ vanish at infinity according to the inverse square law. Since the rod and the coil are oylindrically symmetric, the current flow is assumed to be in coscial circles only: For this case we require:

(2.20) $\hat{A}=\left\{0, A_{\varphi}, 0\right\}$, With

(2.21) $A_{\varphi}=A_{\varphi}(r, u)$.

In a cylindrical coordinate systern $(r, \varphi, u)$ we have (2.22) $\nabla \times(\nabla \times A)=\left\{0^{\prime},-\frac{\partial^{2} A_{\varphi}}{\partial u^{2}}-\frac{\partial^{2} A_{\varphi}}{\partial r^{2}}-\frac{I}{r} \frac{\partial A_{\varphi}}{\partial r}+\frac{A_{\varphi}}{r^{2}} ; 0\right\}$.

By making use of the following definftions:

$(2.23)(a) \quad L_{1}(w)=\left(\frac{\partial^{2}}{\partial u^{2}}+\frac{\partial^{2}}{\partial r^{2}}+\frac{1}{r} \frac{\partial}{\partial r}-\frac{1}{r^{2}}-\frac{\mu^{\prime}}{\mu} \frac{\partial}{\partial r}-\frac{1}{r} \frac{\mu^{\prime}}{\mu}+k^{2}\right) w$ (b) $L_{2}(w)=\left(\frac{\partial^{2}}{\partial u^{2}}+\frac{\partial^{2}}{\partial \mathbf{r}^{2}}+\frac{1}{\mathbf{r}} \frac{\partial}{\partial \mathbf{r}}-\frac{1}{r^{2}}\right) w$,

we derive from $(2.14)$ (a), (b) the differential equation $(2.24)(a) \quad L_{1}\left(A_{\varphi}\right)=0$;

in the rod, and

(2.24)(b) $\quad L_{z}\left(A_{\rho}\right)=0$

in the air. 
From the boundary conditions (2.19) (a) - (c) and the relations (2.12) and (2.13), we derive the boundary conditions at the interface of the rod and the surrounding air to be

$$
\begin{aligned}
& A_{\varphi}^{(r)}=A_{\varphi}^{(c)} \text { for } \quad r \neq R_{0}, \quad|u|<\infty \text {, } \\
& \frac{\partial A_{k}^{(-)}}{\partial u}=\frac{\partial A_{k}^{(a)}}{\partial u} \text { for } r=R_{0}, \quad|u|<\infty \text {, and } \\
& \frac{1}{r} A_{r}^{(r)}+\frac{\partial_{A}^{(H)}}{\partial r}=\frac{\mu}{\mu_{0}}\left(\frac{1}{x} A_{\varphi}^{(a)}+\frac{\partial_{A}^{(a)}}{\partial r}\right) \text { for } r=R_{0},|u|<\infty .
\end{aligned}
$$

Also the boundary conditions between the coil and the surrounding air are

$$
\begin{aligned}
& A_{p}^{(-)}=A_{\varphi}^{(a)} \text { for } r=n_{1} \text { or } R_{2},|u|<\infty \text { : } \\
& \frac{\partial_{A}^{(f)}}{\partial u}=\frac{\partial_{A}^{(a)}}{\partial u} \text { for } r=R_{1} \text { or } R_{p},|u|<\infty, \text { and } \\
& \frac{1}{\mu_{c}}\left(\frac{1}{r} A_{p}^{(c)}+\frac{\partial A_{\psi}^{()}}{\partial r}\right)-\frac{1}{\mu_{0}}\left(\frac{1}{r} A_{p}^{(0)}+\frac{\partial A_{\varphi}^{(0)}}{\partial r}\right)=j_{3 \beta} \\
& \text { for } \mathbf{r}=\mathbf{n} \text {, or } R_{2}, \quad|u|<L \text {. }
\end{aligned}
$$

Since we are studying a problem of surface hardening, the exciting frequency $\omega$ is usually of the order of $10^{6}$ Hertz (cycles per second) or higher and hence the skin depth $\delta=\sqrt{2 / \omega \mu \sigma}[\delta]$ is quite small. If the radius

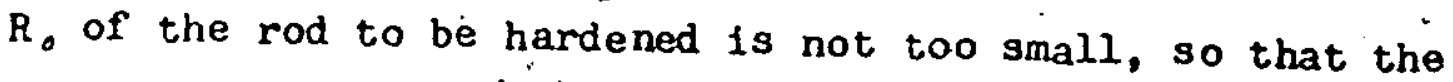
ratio $R_{0} / \delta$ is quite large, then for practicak purposes 
the above boundary value problem can be approximated with sufficient accuracy by a boundary ivalue problem in a halfspace [14],[15]. Geometrically this is equivalent to allowing the radii of the cylinder and coil to becone very large so that their surfaces may be approximated by their tangent planes. In the transition to the limit, these tangent planes remain separated by the distance equal to the difference of the radil, and the metallic cylinder becomes the metallic half-space and the coil becomes an infinite beam aligned parallel to the half space.

- In making the transformation we discard terms containing $I / r^{\circ}$ and $l / r^{2}$ in the differential equation and in the boundary conditions and then introduce the coordinate transf ormation $[14]$ :

$$
\begin{aligned}
(2.25) \quad & x=R_{0}-r, \\
h & =R_{1}-R_{0}, \\
\dot{u} & =u .
\end{aligned}
$$

Also, we consider only a line source of length $\ddot{2} \mathrm{~L}$, instead of a coil, because the coll of finite thickness, $R_{2}-R_{1}>0$, may be obtained from the solution for the line source by superposition, 1 . by integration. The justification for this may be found in $[/ 4]$.

The geometry. of the original boundary value problem is shown in Fig. 2, and thatf of the approximated problem in Fir. 3 . 

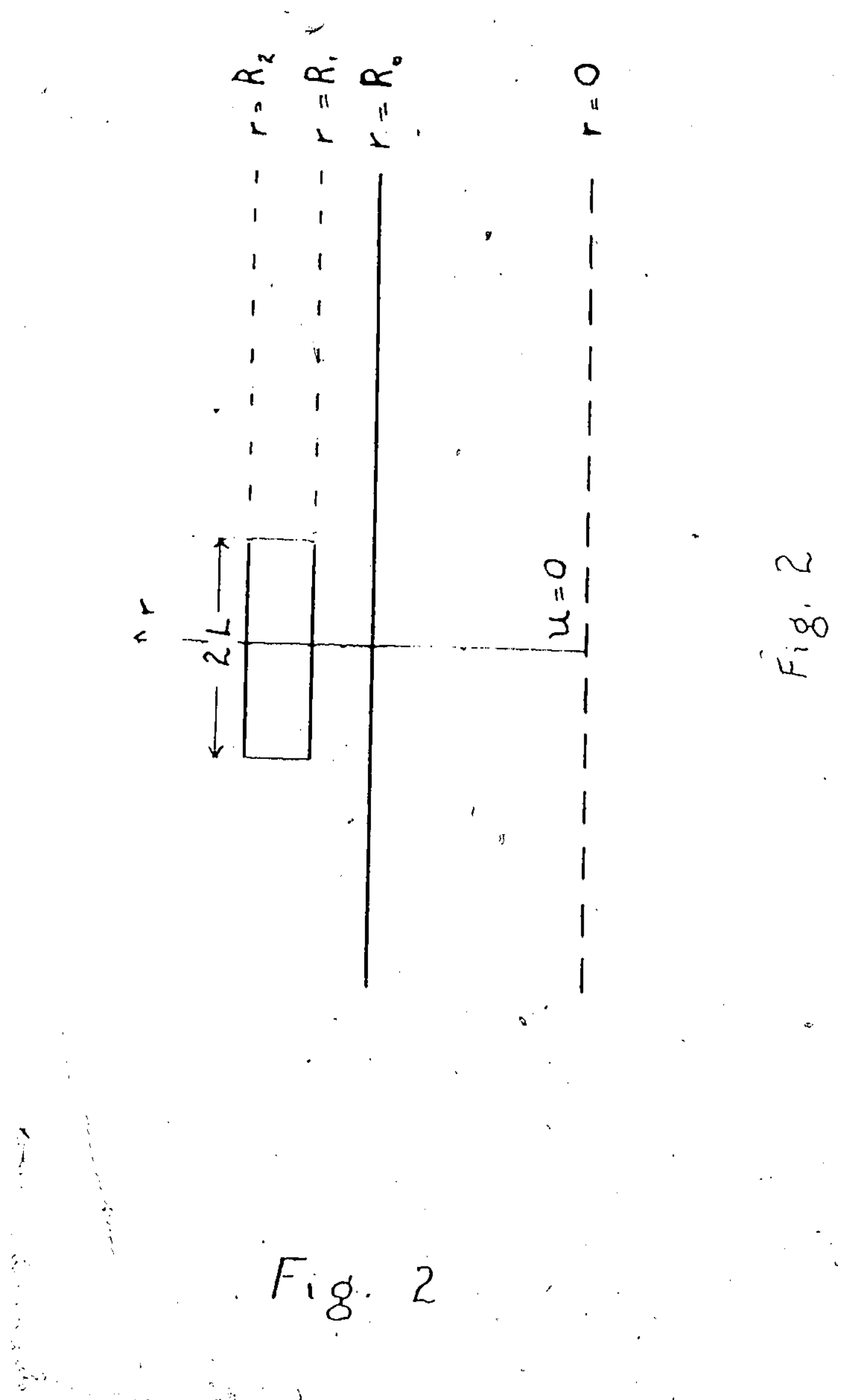

$$
\text { Fig. } 2
$$


z

'


$\begin{array}{lll}\ddots & \\ & 1 & \\ & & \end{array}$

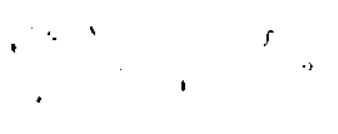

.
J

0

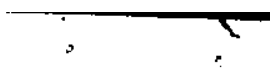

17

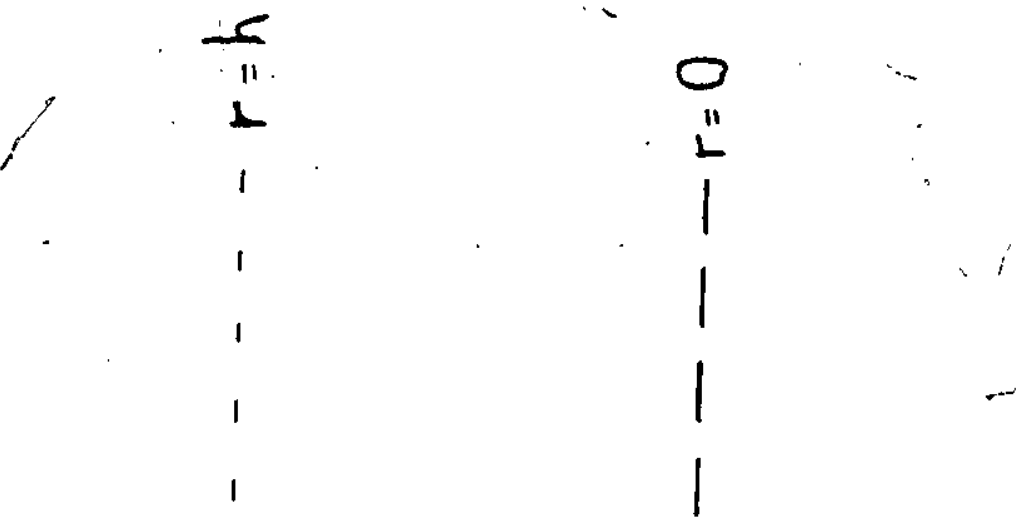


$m$

<smiles>C=CI=CI=CI=C</smiles>

1
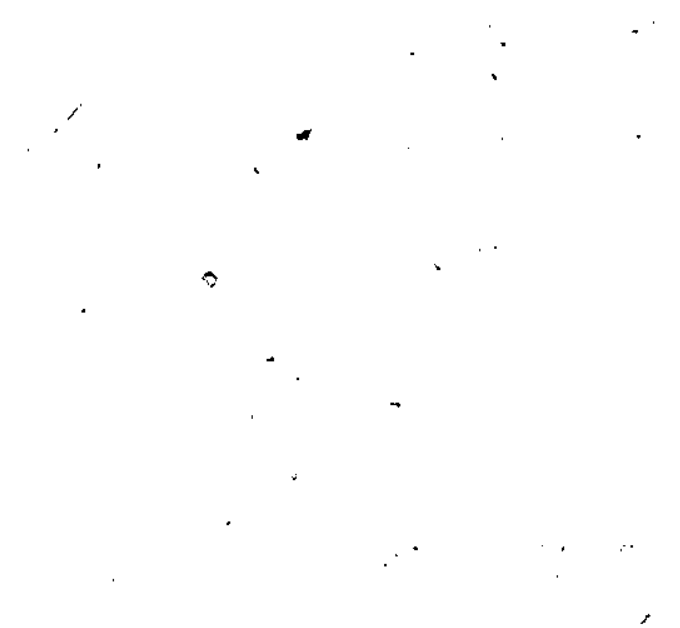
The simplified problem in two dimensions may now be stated thus:

(2.26) $\quad \psi^{(\pi)}=\psi^{(\pi)}(x, u) \quad$ defined on $-\infty<x<-h,|u|<\infty$, $\psi^{(I)}=\psi^{(I)}(x, u)$ defined on $-h<x<0,|u|<\infty$, $\psi=\psi(\ddot{x}, u)$ deflined on $0<x<\infty,|u|<\infty$;

(2.27) $\quad \Delta \dot{\psi}^{(5)}=0$ defined on $-\infty<x<-h,|u|<\infty$,

where $\Delta \psi^{(x)}=\frac{\partial^{2} \psi^{(x)}}{\partial x^{2}}+\frac{\partial^{2} \psi^{(x)}}{\partial u^{2}}$

$(2.28)$ $\Delta \psi^{(n)}=$ o defined on $"-h<x<0,|\dot{u}|<\infty$, (2.29) $\quad \Delta \psi-\frac{\mu^{\prime}(x)}{\mu(x)} \psi_{x}+k^{2}(x) \psi=0, \quad 0<x<\infty,|u|<\infty$, with $k^{2}(x)=-1 \omega \mu(x) \sigma(x)$

The boundary conditions became: $\psi_{x}^{(x)}(-h, u)-\psi_{x}^{(I)}(-h, u)^{\prime}=\left\{\begin{array}{cc}\mu \frac{I}{2 L} & \text { for }|u|<L \\ 0 & \text { for }|u|>L,\end{array}\right.$ $(2.31)(a) \quad \psi^{(\mathbb{I})}(-h, u)-\psi^{(x)}(-h, u)=0$ for $|u|<\infty$ ! (b) $\psi_{u}^{(n)}(-h, u)-\psi_{u}^{(n)}(-h, u)=0$ for $|u|<\infty$, $(2.32)(a) \quad \psi^{(2)}(0, " u)^{\prime}-\psi(0, u)=0 \quad$ for $|u|<\infty$, (b) $\psi_{u}^{(x)}(0, u)-\psi_{k}(0, u)=0$ for $|u|<, \infty$, (c) $\quad \mu(0) \psi_{x}^{(1)}(0, u)-\mu_{0} \psi_{x}(0, u)=0$ for $|u|<\infty$; 
The conditions at infinity are:

$$
\begin{aligned}
& \text { (2.33)(a) } \psi^{(\text {II) }}, \psi^{(\text {I })} \text {, and } \psi \rightarrow 0 \text { as }|u| \rightarrow \infty \text {, } \\
& \text { (b) } \begin{aligned}
\psi^{(x)} & =0\left(|x|^{-2}\right), \text { and } \quad y \\
\psi & =0\left(|x|^{-2}\right) .
\end{aligned}
\end{aligned}
$$

We use the notation $f=O(g(x))$ to signify that $|f| /|g|$ is uniformly bounded as $x \rightarrow \infty$; and $f=o(g(x))$ to signify that $|f| /|g| \rightarrow 0$ as $x \rightarrow \infty$. 
Chapter $\cdot 3$

THE . REPRESENTATION THEOREM

In this chapter we deal with the solution of the boundary value problem formulated in Chapter 2. A representation theorem is proved for the problem.

l. The Statement of the Representation Theorem

The following assumptions are made with regard to the physical parameters $f(x), \sigma(x)$, and $\omega$. for the purposes of the representation theorem. They follow naturally from the physical conditions found in the model.

(A.1) $\dot{\mu}, \sigma$, and $\omega$ are positive.

(A.2) $\mu(x), \sigma(x) \in C^{2}[0, \infty)$.

(A.3) $\omega$ is a congtant.

(A.4) $\underset{x \rightarrow \infty}{\lim } " \mu(x)=\mu_{\infty}$,

$\lim _{x \rightarrow \infty} \sigma_{n}(x)=\sigma_{\infty}$.

(A.5) $\lim _{x \rightarrow \infty} \mu^{\prime}(x)=0$,

$$
\lim _{x \rightarrow \infty} \mu \dot{r}(x)=0
$$


21

$$
\begin{aligned}
& \lim _{x \rightarrow \infty} \sigma^{\prime}(x)=0, \\
& \mu(0)=0 . \\
(A .6) \quad & \int_{0}^{\infty}\left|\mu(x) \sigma(x)-\mu_{\infty} \sigma_{\infty}\right| d x<\infty, \\
& \int_{0}^{\infty}\left|\mu^{\prime}(x)\right|^{2} \mathrm{~d} x<\infty, \text { and }
\end{aligned}
$$

$$
\int_{0}^{\infty}|\mu n(x)| d x<\infty
$$

With these assumptions in mind, the theorem is now stated.

Theorem '(Representation Theorem)

Suppose that $\mu(x), \sigma(x)$, and $\omega$ satisfy assumptions (A.1) to (A.6), then a solution of the boundary value problem "(2.26) - (2.33) exists and can be represented by:

(3.1)

$$
\psi^{(I)}(x, u)=\int_{0}^{\infty}\left[B_{0}(\lambda) \exp \{\lambda x\}+\right.
$$

$$
\left.c_{0}(\lambda) \exp \{-\lambda x\}\right] \cos \lambda u \frac{\sin \lambda L}{\lambda L} d \lambda,
$$

(3.2)

$$
\begin{aligned}
& \text { (3.2) } \psi^{(I)}(x, u)=\int_{0}^{\infty} A_{0}(\lambda) \exp \{\lambda x\} \cos \lambda u \frac{\sin \lambda_{L}}{\lambda L} \\
& \text { (3.3) } \quad \psi(x, u)=\int_{0}^{\infty} A(x, \lambda) \cos \lambda_{u} \frac{\sin \lambda L}{\lambda L} d \lambda,
\end{aligned}
$$$$
\mathbf{d} \lambda \text {, }
$$

where $A_{0}(\lambda), B_{0}(\lambda), C_{0}(\lambda)$, and $A(x, \lambda)$ satisfy the following equations: 




$$
\text { (3.4)(a) } \begin{gathered}
-A_{0}(\lambda) \exp \{-\lambda \mathrm{h}\} \lambda+\mathrm{B}_{0}(\lambda) \exp \{-\lambda \mathrm{h}\} \lambda \\
-c_{0}(\lambda) \exp \{\lambda \mathrm{h}\} \lambda=-\mu \frac{I}{\pi},
\end{gathered}
$$

2 .

$$
\begin{gathered}
A_{0}(\lambda) \exp \{-\lambda h\}-B_{0}(\lambda) \exp \{-\lambda h\} \\
-C_{0}(\lambda) \exp \{\lambda h\}=0 .
\end{gathered}
$$

(c) $-B_{n}(\lambda)-c_{0}(\lambda)+A(0, \lambda)=0$,

(d) $\mu(0) B_{0}(\lambda) \lambda-\mu(0) c_{0}(\lambda) \lambda-\mu_{0} A^{\prime}(0, \lambda)=0$,

(e) $A^{\prime \prime}(x, \lambda)-\frac{\mu^{\prime}(x)}{\mu(x)} A^{\prime}(x, \lambda)$

$+\left[k^{2}(x)-\lambda^{2}\right]_{A}(x, \lambda)=0$,

(f) $A(x, \lambda) \rightarrow 0$ as $x \rightarrow \infty$ for all $\lambda$,

and depotes differentiation with respect to $x$.

4 2. Some Fundamental Lemmas

In order to facilitate the proof of the theorem we first state and prove the following lemmas.

Lemma 1

If $p(x) \in C[0, \infty) \cap L_{1}[0, \infty)$,

then the solution of the differential equation

$$
u(x)+p(x) u(x)=q(x), \quad 0 \leq x<\infty
$$

subject to the Initial condition

$$
\text { (3.6) } u(0)=c \text {, }
$$

when considered as a functional of $q(x)$, is a monotone increasing linear functional of $q(x)$.

Furthermore, the solution of the differential equation (3.5) subject to

$$
\lim _{x \rightarrow \infty} u(x)=c_{2}
$$

is a monotone decreasing Ilnear functional of $q(x)$. 
Proof: $\exp \left\{\int_{0}^{x} p(t) d t\right\}$, we obtain

If wo multiply $(3,5)$ by the integrating factor (3.8) $u^{\prime}(x) \exp \left\{\int_{0}^{x} p(t) d t\right\}+\exp \left\{\int_{0}^{x} p(t) d t\right\} p(x) u(x)=$ $\exp \left\{\int_{0}^{x} p(t) d t\right\} q(x)$

Upon integrating $(3.8)$ and making use of (3.6) wo obtain (3.9) $u(x)=c_{1} \exp \left\{-\int_{0}^{x} p(t) d t\right\}+\exp \left\{-\int_{0}^{x} p(t) d t\right\}$ $\left[\int_{0}^{x} \exp \left\{\int_{0}^{t} p(s) d s\right\} q(t) d t\right]$

From $(3.9)$ it is obvious that $u(x)$ is a linear functional of $g(x)$ and further that the positivity of the exponential function permits us to assert that $u(x)$ is a moriotone increasing functional of $q(x)$.

Similarly, we can show that the solution of (3.5) subject to $(3.7) 18$

(3.10) $u(x)=c_{2} \exp \left\{\int_{x}^{\infty} p(t) d t\right\}-\exp \left\{\int_{x}^{\infty} p(t) d t\right\}$

$$
\left[\int_{x}^{\infty} \exp \left\{-\int_{x}^{\infty} p(s) d s\right\} q(t) d t\right]
$$
and hence, that $u(x)$ is a monotone decreasing functional
of $q(x)$. 
Lemma 2

For $\int_{0}^{\infty}|f(x)| \mathrm{d} x<\infty$ and Real $\alpha>0$, the integral equation

(3.11) $z(x, \propto)=\frac{1}{2 \propto} \int_{0}^{x}[\exp \{\alpha(t-x)\}-\exp \{\alpha(x-t)\}]$

has a unique solution.?

$$
(f(t)[z(t, \propto)+\exp \{\propto t\}] d t) \text {, }
$$

Also, the integral equation .

(3.12) $z(x, \alpha)=\frac{1}{2 \alpha} \int_{x}^{\infty}[\exp \{\alpha(x-t)\}-\exp \{\alpha(t-x)\}]$

$$
(f(t)[z(t, \alpha)+\exp \{-\alpha t\}] d t) \text {, }
$$

has a unique solution.

Proof :

We use the method of successive approximations to solve (3.11). We introduce a sequence of iterates $\left\{z_{n}(x, \propto)\right\}$ defined by $z_{0}(x, \propto)=0$, and for $n \geqslant 1$

$$
\begin{aligned}
z_{n}(x, \alpha)= & \frac{1}{2 \alpha} \int_{0}^{x}[\exp \{\alpha(t-x)\}-\exp \{\alpha(x-t)\}] \\
& \left(f(t)\left[z_{n-1}(t, \alpha)+\exp \{\alpha t\}\right] d t\right) .
\end{aligned}
$$

Since-the variable of integration $t$ is less than $x$ in (3.13) we have 
25

$$
\text { (3.14) } \begin{gathered}
|\exp \{\alpha(2 t-x)\}-\exp \{\alpha x\}| \leq|\exp \{\alpha(2 t-x)\}|+\exp \{\alpha x) \mid \\
=\exp \{\operatorname{Real} \alpha(2 t-x)\}+\exp \{\operatorname{Real} \alpha x)\} \\
\leq 2 \exp \{\operatorname{Real} \alpha x\}
\end{gathered}
$$

For $n=1$ we have

$$
\begin{aligned}
z_{1}(x ; \alpha)= & \frac{1}{2 \alpha} \int_{0}^{x}[\exp \{\alpha(t-x)\}-\exp \{\alpha(x-t)\}] \\
& (f(t) \exp \{\alpha t\} d t) \\
= & \frac{1}{2 \alpha} \int_{0}^{x}(\exp \{\alpha(2 t-x)\}-\exp \{\alpha x\}) f(t) d t,
\end{aligned}
$$

and then by using $(3,14)$ we obtain

(3.15) $\left|z_{1}(x ; \alpha)\right| \leq \frac{\exp \{\operatorname{Real} \alpha x\}}{\operatorname{Real} \alpha} \int_{0}^{x}|f(t)| d t$.

In a similar fashion we obtain

$$
\begin{aligned}
& z_{2}(x, \alpha)-z_{1}(x, \alpha)=\frac{1}{2 \cdot \alpha} \int_{0}^{x}(\exp \{\alpha(t-x)\}-\exp \{\alpha(x-t)\}) \\
& \left(f(t) z_{1}(t, \alpha) d t\right) \\
& =\frac{1}{2 \alpha} \int_{0}^{x}(\operatorname{\theta xp}\{\alpha(2 t-x)\}-\exp \{\alpha x\}) \\
& \left(f(t) \exp \{-\alpha t\} z_{1}(t, \alpha) d t\right) \\
& \left|z_{2}(x, \alpha)-z_{1}(x, \alpha)\right| \leqslant \frac{\exp \{\operatorname{Real} \alpha x\}}{\operatorname{Real} \alpha} \text {. } \\
& \int_{0}^{\dot{x}}|f(t)| \exp \{-\operatorname{Real} \alpha t\} \cdot\left|z_{1}(t, \alpha)\right| d t
\end{aligned}
$$




$$
\begin{aligned}
& \leqslant \frac{\exp \{\operatorname{Real} \alpha x\}}{\operatorname{Real} \alpha} \int_{0}^{x}|f(t)| \frac{\int_{0}^{t}|f(s)| / d s}{\operatorname{Re} a l \alpha} d t \\
& =\frac{\exp \{\operatorname{Real} \alpha x\}}{(\operatorname{Real} \alpha)^{2}} \int_{0}^{x} \frac{d}{d t} \frac{\left[\int_{0}^{t} \cdot|f(s)| d \dot{s}\right]^{2}}{2} d t \\
& =\exp \{\operatorname{Real} \dot{\alpha} x\}\left[\frac{\int_{0}^{t}|f(t)| d t}{\operatorname{Real}}\right]^{2} \\
& 2
\end{aligned}
$$


(3.16) $\left|z_{n}(x, \alpha)-z_{n-1}(x, \alpha)\right| \leq \frac{\exp \{\operatorname{Real} \alpha x\}}{n !}\left[\frac{\int_{0}^{x}|f(t)| d t}{\operatorname{Real} \alpha}\right]^{n}$. Since $z_{n+1}(x, \alpha)$ may be written as a series in the form (3.17) $z_{n+1}(x, \alpha)=\sum_{i=1}^{n}\left[z_{i+1}(x, \alpha)-z_{i}(x, \alpha)\right]$, the convergence of the sequence $\left\{z_{n}(x, \alpha)\right\}$ to a limit function $\mathrm{z}(\mathrm{x}, \alpha)$ as $\mathrm{n} \rightarrow \infty$ is equivalent to the convergence of the series (3.I7). However, it is obvious that the series (3.17) is also absolutely convergent because by (3.16) each term in (3.17) is less in absolute value than $\exp \{$ Real $\propto x\}$ times a term in the convergent series for $\exp \left\{\frac{\int_{0}^{x}|f(t)| d t}{\text { Real } \alpha}\right\}:$ Thus the sequence of iterates $\left\{z_{n}(x, \alpha)\right\}$ converges to a limit function $z(x, \alpha)$ uniformly in every finite subinterval of $[0, \infty)$. Thus we now have 
the solution of the integral equation $(3.11)$ by the method of successive approximations. It remains to be shown that the solution for (3.11) is unique.

To show uniqueness we suppose instead that there exist two solutions $z^{(1)}(x, \alpha)$ and $z^{(2)}(x, \alpha)$ satisfying. $(3.11)$. We then have

$$
\begin{aligned}
& z^{(k)}(x, \alpha)-z^{(1)}(x, \alpha)=\frac{1}{2 \alpha} \int_{0}^{x}[\exp \{\alpha(t-x)\}-\exp \{\alpha(x-t)\}] \\
& \left(f(t)\left[z^{(2)}(x ; \alpha)-z^{n}(x, \alpha)\right] d t\right) \\
& =\frac{1}{2 \alpha} \int_{0}^{x}[\exp \{\alpha(2 t-x)\}-\exp \{\alpha x\}] \\
& (f(t) \exp \{-\alpha t\}) \\
& {\left[z^{(2)}(t, \alpha)-z^{(1)}(t, \alpha)\right] d t} \\
& \left|z^{(2)}(x, \alpha)-z^{(t)}(x, \alpha)\right| \leq \frac{\exp \{\operatorname{Real} \alpha x\}}{\operatorname{Real} \alpha} \int_{0}^{x}|f(t)| \exp \{- \text { Real } \alpha t\} \\
& \left|z^{(z)}(t, \alpha)-z^{(1)}(t, \alpha)\right| d t \\
& \text { (3.18) exp }\{-\operatorname{Real} \alpha x\}\left|z^{(2)}(x, \alpha)-z^{(1)}(x, \alpha)\right| \\
& \leqslant \int_{0}^{x} \frac{|f(t)|}{\operatorname{Real} \alpha} \exp \{-\operatorname{Real} \alpha t\} \\
& \left|z^{(2)}(t, \alpha)-z^{(1)}(t ; \alpha)\right| d t \text {. }
\end{aligned}
$$

If we put:

(3.19) $|g(x, \alpha)|=\exp \{-\operatorname{Real} \alpha x\} \cdot z^{(2)}(x, \alpha)-z^{(1)}(x, \alpha) \mid$ wo have from (3.18) 
(3.20) $\quad|g(x, \alpha)| \leqslant \int_{0}^{x} \frac{|f(t)|}{\operatorname{Real} \alpha}|g(t, \alpha)| d t$.

If we can show that the solution of this integral inequality is fust the trivial solution, then by (3.19) we have $z^{(2)}(x, \alpha) \equiv z^{(1)}(x, \alpha)$. In order to show this we let

$(3.2 i) \quad h_{1}(x, \alpha)=\int_{0}^{x} \frac{|f(t)|}{\operatorname{Real} \alpha}|g(t, \alpha)| d t$ From (3.20) we have. (3.22) $\quad|g(x, \alpha)| \leq h_{1}(x, \alpha)$. Differentiating (3.21) with respect to $x$ we obtain

$$
\begin{aligned}
h_{1} \cdot(x, x) & =\frac{|f(x)|}{\operatorname{Real} \alpha}|g(x, x)| \\
& \leqslant \frac{|f(x)|}{\operatorname{Real} \alpha} \mathrm{h}_{1}(x, \propto)
\end{aligned}
$$

and thus deduce that

(3.23) (a) $h_{1} \cdot(x, \alpha)-\frac{|f(x)|}{\operatorname{Real} \alpha} \quad h_{1}(x, \alpha) \leqslant 0$

while from $(3.21)$ we obtain.

$(3,23)(b) \quad h,(0, \alpha)=0$.

Now, if we compare (3.23)(a) and (b) with the following differential equation 6: (3.24)(a) $\quad h_{2}^{\prime}(x, \alpha)-\frac{|f(x)|}{\operatorname{Real} \alpha} h_{2}(x, \alpha)=0$ subject to $(3.24)(b) \quad h_{z}(0, \alpha)=0$, 
and apply Lemma $I$ and $[1]$ p. 134 , we conclude that

$$
\text { (3.25) } \quad h_{1}(x, \infty) \leqslant h_{2}(x, \alpha) \text {. }
$$

Howe ver, the solution $h_{2}(x, \alpha)$ of $(3.24)(a)$ and (b)' is fust the trivial solution. Hence by (3.22) and (3.25) we have $|g(x, \alpha)|=0$.

Similarly we" can, employ the method of successive approximations to prove that (3.12) has a unique solution.

Lemma 3

For $\int_{0}^{\infty}|f(x)| d x<\infty$ and Real $\alpha>0$, the differential equation

$$
\text { (3.26) } \quad Y^{n}(x, \alpha)-\left[\alpha^{2}-f(x)\right] Y(x, \alpha)=0
$$

has two linearly independent solutions for all $0 \leq x<\infty$, and for all $\propto$ with Real $\propto>0$, and these a re given by (3.27) (a) $Y_{1}(x, \dot{\alpha})=\exp \{\alpha x\}\left[1+b_{1}(x, \alpha)\right]$, and (b) $\quad Y_{2}(x, \alpha)=\exp \{-\alpha x\}\left[1+b_{2}(x, \alpha)\right]$, with $(3.28)(a) \quad\left|b_{1}(x, \alpha)\right| \leqslant \exp \left\{\frac{\int_{0}^{x}|f(t)| d t}{\operatorname{Real} \alpha}\right\}-1, \quad$ and (b) $\left|b_{2}(x, \alpha)\right| \leqslant \exp \left\{\frac{\int_{x}^{\infty}|f(t)| d t}{\operatorname{Real} \alpha}\right\}-1$.

Proof :

Let

$(3.29)(a) \quad z(x, \alpha)=\exp \{\alpha x\} b_{1}(x, \alpha)$. 
so that

$(3,29)(b) . \quad Y_{1}(x, \alpha)=\exp \{\alpha x\}+z(x, \alpha)$.

Substituting (3.29)(b) into (3.26) we obtain

(3.30) $\quad z^{\prime \prime}(x, \alpha)-\alpha^{2} z(x, \ddot{\alpha})=-f(x)[z(x, \alpha)+\exp \{\alpha x\}$. Now (3.30) may be transformed into the integral equation (3.1.1). By Lemma 2 the solution to this integral equation exists and hence $Y_{1}(x, \dot{\alpha})$ exists.

It remains to show that $b,(x, x)$ satisfies the oatimate $(3,28)(a)$.

Frnm $(3.16)$ and $(3.17)$ we obtain

$(7.31)$

$$
\begin{aligned}
|r(x, \alpha)| & \leq \sum_{j=0}^{\infty}\left|z_{j, 1}(x, \alpha)-z_{j}(x, \alpha)\right| \\
& \leq \exp \left\{\operatorname{Re} 1 \alpha x \mid\left[\exp \left\{\frac{\int_{0}^{x}|f(t)| d t}{\operatorname{Real} \alpha x}\right\}-1\right] .\right.
\end{aligned}
$$

loing $(7.29)(a)$ and $(3.31)$ wo obtain

$$
\begin{aligned}
\left|r_{i}(x, \mu)\right| & \leqslant \exp \{-\operatorname{Rëal} \alpha x\}|z(x, \alpha)| \\
& \leqslant \exp \left\{\frac{\int_{0}^{x}|f(t)| d t}{\operatorname{Real} \alpha}\right\}-1 .
\end{aligned}
$$

Similarly we can show that $\mathrm{Y}_{2}(x, \alpha)$ exists and that $b_{2}(x, \alpha)$ satisfies the estimate $(3.28)(b)$.

Lemma 4

There exists a unique solution for the differential equation $(3.4)(e)$ subject to $(3.4)(f)$ and the following condition (3.32):

$$
\mu(0) \lambda_{A}(0, \lambda)-\mu_{0} A^{\prime}(0, \lambda)=\frac{\mu_{0} \mu(0)}{\pi} I \exp \left\{-\lambda_{h}\right\}
$$


Proof :

4

We, first note that (3.32) is obtained by eliminating $A_{0}(\lambda), B_{0}(\lambda)$, and $C_{0}(\lambda)$ from equations $(3.4)(a)-(d)$. If we introduce the one to one transformation:

$$
(3.33)^{-x} \quad \mathbf{a}(x, \lambda)=\sqrt{\frac{\mu(0)}{\mu(\sqrt{x})}} \cdot A(x ; \lambda)
$$

then equation $(3.4)(e)$ is reduced to

$$
a^{n}(x, \lambda)-\left[\lambda^{2}-K^{\prime}(x)\right] a(x, \lambda)=0
$$

where

$$
k^{2}(x)+k^{2}(x)-\frac{3}{4}\left[\frac{\mu \cdot(x)}{\mu(x)}\right]+\frac{1}{2} \frac{\mu^{n}(x)}{\mu(x)} .
$$

By (A.5) and (3.33) we also have:

$$
a(0, \lambda)=A(0, \lambda) \text {, }
$$

(b)

$$
a^{\prime}(0, \lambda)=A^{\prime}(0, \lambda) \text {. }
$$

Substitution of $(3.36)(a)$ and (b) into (3.32) and substitution of $(3.33)$ into $(3.4)(f)$ ytelds:

$$
(3.37)(a)
$$

$$
\begin{aligned}
& \mu(0) \lambda \mathrm{a}\left(0, \lambda_{1}\right)-\mu_{0} a \cdot(0, \lambda) \\
& =\mu_{0} \frac{\mu(0)}{\pi} I \exp \{-\lambda h\}, \text { and }
\end{aligned}
$$

(b) a $(x, \lambda) \rightarrow 0$ as $x \rightarrow \infty$, for all $\lambda$. Hence if we can prove that a solution exists for $(3.34)$ subject to conditions $(3.37)(a)$ and (b) and that this solution is unique, then this is also true for $A(x, \lambda)$.

Let us then define

$(3.38)(a)$

$$
\mathrm{K}_{\infty}^{2}=\lim _{\mathrm{x} \rightarrow \infty} \mathrm{K}^{2}(\mathrm{x})
$$


then by $(A .5)$ we have

$(3.38)(b), K_{\infty}^{2}=k_{\infty}^{2}=-1 \omega \mu_{\infty} \sigma_{\infty}$.

and hence.

(3.39) $\sqrt{\lambda^{2}-K_{\infty}^{2}}=\frac{1}{\sqrt{2}} \sqrt{\sqrt{\lambda^{+}+\omega^{2} \mu_{\infty}^{2} \sigma_{\infty}^{2}}+\lambda^{2}}$

Also from (3.35), (3.38) and (A.1) - (A.6) we obtain (3.40) $\int_{0}^{\infty}\left|\mathrm{K}^{2}(x)-\mathrm{K}_{\infty}^{2}\right| \mathrm{d} x<\infty$.

Now equation $(3.34)$ may be rewritten as

$$
\mathrm{a}^{n}(\mathrm{x}, \lambda)-\left\{\left(\lambda^{2}-\mathrm{k}_{\infty}^{2}\right)-\left[\mathrm{K}^{2}(\mathrm{x})-\mathrm{K}_{\infty}^{2}\right]\right\} \mathrm{a}(\mathrm{x} ; \lambda)=0
$$

hence the assumptions of Lemma 3 are satisfied by (3.34). Then by Lemma 3 there are two linearly independent solutions $a_{1}\left(x, \lambda\right.$ and $a_{2}(x, \lambda)$ to $(3.34)$ such that for all $0 \leqslant x<\infty$ and $o \circ>\lambda \geqslant 0$ it holds that:

$$
\begin{aligned}
(3.41)(a) \quad \therefore a_{1}(x, \lambda) & =\exp \left\{\sqrt{\lambda^{2}-k_{\infty}^{2}} x\right\}\left[1+d_{1}(x, \lambda)\right] \\
\text { (b) } \quad a_{2}(x, \lambda) & =\exp \left\{-\sqrt{\lambda^{2}-k_{\infty}^{2}} x\right\}\left[1+d_{2}(x, \lambda)\right]
\end{aligned}
$$

$\because(3.42)(a) \quad\left|d_{1}(x, \lambda)\right| \leq \exp \left\{\frac{\int_{0}^{x}\left|K^{2}(t)-x_{\infty}^{2}\right| d t}{\operatorname{Real} \sqrt{\lambda^{2}-K_{\infty}^{2}}}\right\}-1$,

(b)

$$
\left|d_{2}(x, \lambda)\right| \leqslant \exp \left\{\frac{\int^{\infty}\left|k^{2}(t)-k_{\infty}^{2}\right| d t}{\operatorname{Real} \sqrt{\lambda^{2}-k_{\infty}^{2}}}\right\}-1
$$

Now from: $(3.41)(a)$ and (b) and from (3.42)(a) and (b) we have for large values of $x$ and for all values $0 \leqslant \lambda<\infty$ 
(3.43)(a) $\quad a_{1}(x, \lambda)=\exp \left\{\sqrt{\lambda^{2}-K_{\infty}^{2}} x\right\}^{\prime}[1+0(1)]$

(b) $\quad a_{2}(x, \lambda)=\exp \left\{-\sqrt{\lambda^{2}-K_{\infty}^{2}} x\right\}[1+0(1)]$.

Since $(3.34)^{\prime \prime}$ is linear we have

$$
a\left(x, \lambda r=c_{1}(\lambda) a_{1}(x, \lambda)+c_{2}(\lambda) a_{2}(x, \lambda)\right.
$$

for all $0 \leqslant x<" \infty$ and for all $0 \leqslant \lambda<\infty$.

In particular for large values of $x$ we have

$$
\begin{aligned}
a(x, \lambda)= & c_{1}(\lambda) \exp \left\{\sqrt{\lambda^{2}-K_{\omega}^{2}} \cdot x[1]+0(1)\right\} \\
& +c_{2}(\lambda) \exp \left\{-\sqrt{\lambda^{2}-K_{\infty}^{2}} x\right\}[1+0(1)] .
\end{aligned}
$$

From (3.37) (b) we see that in order for $a(x, \lambda) \rightarrow 0$ as $x \rightarrow \infty$ for all $0 \leqslant \lambda<\infty$, we must have $c,(\lambda)=0$

for all $0 \leqslant \lambda<\infty$. Therefore we have (3.44). $a(x, \lambda)=c_{2}(\lambda) \cdot a_{2}(x, \lambda)$.

If we substitute $(3.44)$ into $(3.34)(c)$ we obtain

$$
c_{2}(\lambda)=\frac{\mu_{0}(\mu(0) / \pi) I \exp \{-\lambda h\}}{\mu(0) \lambda a_{2}(0, \lambda)-\mu_{0} a_{2}^{\prime}(0, \lambda)}
$$

for all $0 \leqslant \lambda<\infty$. Hence we have:

(3.45) $\quad a(x, \lambda)=\frac{\mu_{0}(\mu(0) / \pi) I \exp \{-\lambda \mathrm{h}\}}{\mu(0) \lambda \mathrm{a}_{2}(0, \lambda)-\mu_{0} \mathrm{a}_{2}(0, \lambda)} \mathrm{a}_{2}(x, \lambda)$.

From (3.33) we deduce that

$$
\text { (3.46) } \begin{aligned}
A(x, \lambda) & =\sqrt{\frac{\mu(x)}{\mu(0)}} a(x, \lambda) \\
& =\sqrt{\frac{\mu(x)}{\mu(0)}}\left[\frac{\mu_{0}(\mu(0) / \pi) I \exp \{-\lambda h\}}{\mu(0) \lambda a_{i}(0, \lambda)-\mu_{0} a_{2} \cdot(0, \lambda)}\right] a(x, \lambda)
\end{aligned}
$$


Thus we have shown the existence and uniqueness of the solution to $(3.4)(c)$ subject to $(3.4)(f)$ and $(3.32)$. As a corollary to Lemma 4 we obtain the behaviour of $A(x, \lambda)$ for large values of $x$ and for all values of $0 \leq \lambda<, \infty$.

Corollary to Lemma 4

For large values of $x, A(x, \lambda)$ behaves as $\exp \left\{-\sqrt{\lambda^{2}-k_{\infty}^{2}} x\right\}$.

Proof :

From (3.43)(b) we have for all values of $0 \leqslant \lambda<\infty$ and for large $x$, that $a_{2}(x, \lambda)$ behaves as exp $\left\{\sqrt{\lambda^{2}-K_{\infty}^{2}} x\right\}$. Hence, from $(3.46)$, we conclude that $A(x, \lambda)$ has the same behaviour.

Lemma 5

For large values of $\lambda$ and for all values of $0 \leq x<, \infty$ then there exist solutions to the Ricatt equations

(3.47)(a) $\phi^{\prime}(x, \lambda)+\phi^{2}(x, \lambda)=\lambda^{2}-k^{2}(x)$

subject to

$(3.47)(b) \quad \phi(x, \lambda) \rightarrow-\sqrt{\lambda^{2}-k_{\infty}^{2}} \quad$ as $x \rightarrow \infty$.

Proof :

If we let $w(x, \lambda)=\emptyset(x, \lambda))_{0}+\sqrt{\lambda^{2}-k_{\infty}^{2}}$ and substitute this into equation (3.47) (a) and (b) we obtain: : 


$$
\begin{aligned}
& \text { (3.48)(a) w! }(x, \lambda)+w^{2}(x, \lambda)-2 \sqrt{\lambda^{2}-k_{\infty}^{2}} w(x, \lambda) \\
& =\left[\mathrm{K}_{\infty}^{2}-\mathrm{K}^{2}(\mathrm{x})\right] \text {, } \\
& \text { (b) } w(x, \lambda) \rightarrow 0 \text { as } x \rightarrow \infty \text {. }
\end{aligned}
$$

From $(3.38)(a)$ we deduce that $\lim _{x \rightarrow \infty}\left|K^{2}(x)-K_{\infty}^{2}\right|=0$ and from assumption (A.2) we conclude that $\left|K^{2}(x)-K_{\infty}^{2}\right|$ $\epsilon_{0} c[0, \infty)$; and hence that $\left|K^{2}(x)-K_{x}^{2}\right|$ is uniformly bounded. Now let $M$ be such a bound, then we have (3.49)

$$
\left|K^{2}(x)-K_{\infty}^{2}\right|<M \text { for all } 0 \leqslant x<\infty \text {. }
$$

From (3.39) we observe that Real $\sqrt{\lambda^{2}-K_{\infty}^{2}}$ is a positive monotone increasing function of $\lambda$, hence there exists" a $\lambda_{0}$ such that for all $\lambda \geqslant \lambda_{0}$ : (3.50) $\operatorname{Re} \sqrt{\lambda^{2}-K_{\infty}^{2}}>1+M$.

Now equation $(3.48)$ (a) may be reduced to the following integral equation:

(3.5I) $w(x, \lambda)=c(\lambda) \exp \left\{2 \sqrt{\lambda^{2}-K_{x}^{2}} x\right\}+\exp \left\{\sqrt[2]{\lambda^{2}-K_{\infty}^{2}} x\right\}$

$$
\int_{0}^{x} \exp \left\{-2 \sqrt{\lambda^{2}-k_{\alpha}^{2}} \mathbf{s}\right\} \text {. }
$$

$$
\left[\mathrm{K}_{\infty}^{2}-\mathrm{K}^{2}(\mathrm{~s})-\mathrm{w}^{2}(\mathrm{~s}, \lambda)\right] \mathrm{ds},
$$

where $c(\lambda)$ is an arbitrary constant which depends on the parameter $\lambda$. The presence of $\exp \left\{2 \sqrt{\lambda^{2}-K_{\infty}^{2}} x\right\}$ can. lead to difficulty, so we rewrite the equation as 
$(3.52)$

$$
\begin{aligned}
w(x, \dot{\lambda})= & c(\lambda) \exp \left\{2 \sqrt{\lambda^{2}-K_{\infty}^{2}} x\right\} \\
& +\exp \left\{\sqrt[2]{\lambda^{2}-K_{\infty}^{2}} x\right\} \int_{0}^{\infty} \exp \left\{-2 \sqrt{\lambda^{2}-K_{\infty}^{2}} s\right\} \\
& {\left[K_{\infty}^{2}-K^{2}(s)-w^{2}(s, \lambda)\right] d s } \\
& -\exp \left\{2 \sqrt{\lambda^{2}-K_{\infty}^{2}} x\right\} \int_{x}^{\infty} \exp \left\{-2 \sqrt{\lambda^{2}-K_{\infty}^{2}} s\right\} \\
& {\left[K_{\infty}^{2}-K^{2}(s)-w^{2}(x, \lambda)\right] d s . }
\end{aligned}
$$

In onder that $w(x, \lambda)$ shall satisfy $(3.48)(b)$

the $c(\lambda)$ must satisfy

$$
c(\lambda)=-\int_{0}^{\infty} \exp \left\{-2 \sqrt{\lambda^{2}-K_{\infty}^{2}} s\right\}\left[k_{\infty}^{2}-k^{2}(s)-\bar{w}^{2}(s, \lambda)\right] d s .
$$

Hence $(3.52)$ reduces to the following:

$$
\begin{aligned}
w(x, \lambda)= & \exp \left\{2 \sqrt{\lambda^{2}-\mathrm{K}_{\infty}^{2}} x\right\} \int_{x}^{\infty} \exp \left\{-2 \sqrt{\lambda^{2}-\mathrm{K}_{\infty}^{2}} \mathrm{~s}\right\}, \\
& {\left[\mathrm{w}^{2}(\mathrm{~s}, \lambda)-\mathrm{K}^{2}(\mathrm{~s})-\mathrm{K}_{\infty}^{2}\right] \mathrm{ds} . }
\end{aligned}
$$

We now apply the method of successive approximations -

([2] pp. 29-30) with

$(3.54)(a) \quad w_{0}(x, \lambda)=\exp \left\{2 \sqrt{\lambda^{2}-K_{\infty}^{2}} x\right\} \int_{x}^{\infty} \exp \left\{-2 \sqrt{\lambda^{2}-K_{\infty}^{2}} s\right\}$.

$$
\left[\mathrm{K}^{2}(\mathrm{~s})-\mathrm{K}_{\infty}^{2}\right] \mathrm{ds}
$$

(b)

$$
w_{n+1}(x, \lambda)=\exp \left\{2 \sqrt{\lambda^{2}-k_{\infty}^{2}} x\right\}
$$

$$
\begin{array}{r}
\int_{x}^{\infty} \exp \left\{-2 \sqrt{\lambda^{2}-k_{\infty}^{2}} s\right\} \\
{\left[w_{n}^{2}(s, \lambda)+k^{2}(s)-k_{\infty}^{2}\right] d s}
\end{array}
$$

for $n \geqslant 0$. We next wish to show that this iteration 
37

converges for all $0 \leqslant x<\infty$ and for all $-\lambda \geqslant \lambda_{0}$.

Wo first show by induction that for all $0 \leq x<\infty$ and for all $\lambda_{0}: \leqslant<<\infty$ :

(3.55). $\quad\left|w_{n}(x, \lambda)\right|<\frac{M}{1+M}<1$. for all $n \geqslant 0$.

From $(3.54)(a)$ we observe that
\[ \left|w_{0}(x, \lambda)\right| \leqslant \exp \left\{2 \operatorname{Re} \sqrt{\lambda^{2}-K_{\infty}^{2}} x\right\} \int_{x}^{\infty} \exp \left\{-2 \operatorname{Re} \sqrt{\lambda^{2}-K_{\infty}^{2}} 8\right\} \]

$$
\left|K^{2}(s)-K_{\infty}^{2}\right| d s
$$

$\leq M \exp \left\{2 \operatorname{Re} \sqrt{\lambda^{2}-K_{\infty}^{2}} x\right\}$

$$
\begin{aligned}
& \int_{x}^{\infty} \exp \left\{-2 \operatorname{Re} \sqrt{\lambda^{2}-K_{\infty}^{2}} s\right\} d s . \\
= & \frac{M}{2 R \theta \sqrt{\lambda^{2}-K_{\infty}^{2}}}<\frac{M}{2(1+M)}<\frac{M}{1+M}<1 .
\end{aligned}
$$

If we assume that the relation holds for $n$, it then follows from (3.54) (b) that.

$$
\begin{aligned}
\left|w_{n+1}(x, \lambda)\right| \leqslant & \exp \left\{2 \operatorname{Re} \sqrt{\lambda^{2}-K_{\infty}^{2}} x\right\} \\
& \int_{x}^{\infty} \exp \left\{-2 \operatorname{Re} \sqrt{\lambda^{2}-K_{\infty}^{2}} s\right\} \\
& \left|w_{n}^{2}(s, \lambda)\right| d s+\left|w_{0}(x, \lambda)\right| \\
\leqslant & \left(\frac{M}{I+M}\right)^{2} \exp \left\{2 \operatorname{Re} \sqrt{\lambda^{2}-K_{\infty}^{2}} x\right\} \\
& \int_{x}^{\infty} \exp \left\{-2 \operatorname{Re} \sqrt{\lambda^{2}-K_{\infty}^{2}} s\right\} d s+\left|w_{0}(x, \lambda)\right|
\end{aligned}
$$


38

$$
\begin{aligned}
& \leq\left(\frac{M}{1+M}\right)^{2} \frac{1}{2 \operatorname{Re} \sqrt{\lambda^{2}-K_{\infty}^{2}}}+\frac{M}{2(1+M)^{\prime}} \\
& \leq\left(\frac{M}{1+M}\right)^{2} \frac{1}{2(1+M)}+\frac{M}{2(1+M)} \\
\& & \leq\left(\frac{M}{1+M}\right)^{2} \frac{1}{2}+\frac{M}{2(1+M)} \\
& =\left(\frac{M}{1+M}\right)\left(\frac{1}{2} \frac{M}{1+M}+\frac{1}{2}\right)<\frac{M}{1+M}
\end{aligned}
$$

since $M /(1+M)<1$ we have that:

$$
\begin{aligned}
& (3.56)^{\prime}\left|w_{n+1}\left(x, \lambda_{1}\right)-w_{n}(x, \lambda)\right| \leqslant \exp \left\{2 \mathrm{Re} \sqrt{\lambda^{2}-\mathrm{K}_{\infty}^{2}} x\right\} \\
& \int_{x}^{\infty} \exp \left\{-2 \cdot \operatorname{Re} \sqrt{\lambda^{2}-K_{\infty}^{2}} s\right\}\left|w_{n}(s, \lambda),-\dot{w}_{n-1}(s, \lambda)\right| \\
& \left|w_{n}(s, \lambda)+w_{n-1}(s, \lambda)\right| d s \\
& \leq 2 \frac{M}{1+M} \exp \left\{2 \operatorname{Re} \sqrt{\lambda^{2}-K_{r}^{2}} x\right\} \text {. } \\
& \int_{x}^{\infty} \exp \left\{-2 \operatorname{Re} \sqrt{\lambda^{2}-K_{\infty}^{2}} \mathrm{~s}\right\} \text {. } \\
& \left|w_{n}(s, \lambda)-w_{n-1}(s, \lambda)\right| \text { dst. }
\end{aligned}
$$

Furthermore, for $\mathrm{n}=1$ we have

$$
\begin{aligned}
& \left|w_{1}(x, \lambda)-w_{0}(x, \lambda)\right| \leqslant \exp \left\{2 \operatorname{Re} \sqrt{\lambda^{2}-K_{\infty}^{2}} x\right\} \\
& \int_{x}^{\infty} \exp \left\{-2 \operatorname{Re} \sqrt{\lambda^{2}-K_{\infty}^{2}} s\right\}\left|w_{0}^{2}(s, \lambda)\right| d s \\
& \leq \\
& \int_{x}^{\infty} \exp \left\{-2 \operatorname{Re} \sqrt{\lambda^{2}-K_{\infty}^{2}} s\right\} d s
\end{aligned}
$$



4 


$$
\begin{aligned}
& =\left(\frac{M}{1+M}\right)^{2} \frac{1}{2 R \theta \sqrt{\lambda^{2}-K_{\infty}^{2}}} \\
& \leq\left(\frac{M}{1+M}\right)^{2} \frac{i}{2(1+M)}<\frac{1}{2}\left(\frac{M}{1+M}\right)^{2}
\end{aligned}
$$

Iterating this relation in $(3.52)$ we obtain

$$
\text { (3.57) }\left|w_{n+1}(x, \lambda)-w_{n}(x, \lambda)\right| \frac{1}{2}\left(\frac{M}{I+M}\right)^{n+2} \text {. }
$$

Hence the series

$$
\sum_{n=0}^{\infty}\left|w_{n+1}(x, \lambda)-w_{n}(x, \lambda)\right| \leqslant \sum_{n=0}^{\infty} \frac{1}{2}\left(\frac{M}{1+M}\right)^{2+n}=\frac{1}{2} \frac{M^{2}}{1+M}
$$

converges uniformly for all $0 \leqslant x<\infty$ and for all $\lambda \geqslant \lambda_{0}$ to a function $w(x, \lambda)$ which satisfies the integral equation (3.53) and hence satisfies the Ricatti equations.

Lemma 6

For large values of $\lambda ; A(x, \lambda)$ behaves as

$A(0, \lambda) \exp \{-\lambda x\}$.

Proof:

We introduce the transformation:

(3.58) $y(x, \lambda)=A(0, \lambda) \sqrt{\frac{\mu(x)}{\mu(0)}} \exp \left\{\int_{0}^{x} \phi(x, \lambda) d x\right\}$

which we substitute into $(3.4)(\mathrm{e})$ to obtain $(3.47)(\mathrm{a})$. From the corollary to Lemma 4 , wo have that $A(x, \lambda)$ behaves as $\exp \left\{-\sqrt{\lambda^{2}-K_{\infty}^{2}} x\right\}$ for large values of $x$, and hence we obtain conditions $(3.47)(b)$. 
Further, if we introduce the transformation:

(3.59) $\cdot I(x, \lambda)=(1 / \lambda) \not(x, \lambda)$

then (3.47)(a) and (b) are transformed into:

(3.60)(a) $\quad \lambda^{\prime}(x, \lambda)+\lambda^{2} \Phi^{2}(x, \lambda)-\lambda^{2}+K^{2}(x)=0$,

(b) $\quad \Psi(x, \lambda) \rightarrow-(1 / \lambda) \sqrt{\lambda^{2}-K_{\infty}^{2}}$ as $x \rightarrow \infty$.

It has been shown in [?] p. 191, in [3], and in [2] that for large values of $\lambda$, we have :

$$
\Phi(x, \lambda)=\left[-1+0\left(1 / \lambda^{2}\right)\right] \text { for all } 0^{\circ} \leq x<\infty .
$$

from $(2.47)$ we deduce that

$$
\Phi(x, \lambda)=\lambda\left[-1+o\left(1 / \lambda^{2}\right)\right] \text {. }
$$

If we can use (3.62) in (3.58) we conclude that $A(x, \lambda)$ hehaves as $A(0, \lambda) \exp \{-\lambda x\}$, which proves the lemma.

l, emma 7

Let $f(x) \in L_{1}(0 ; \infty), F(x)$ be defined and uniformly bounded on $(-\infty, \infty)$, and for large values of $|t|$,

$$
\int_{0}^{t} F(x) d x=o(t)
$$

then $\lim _{|t| \rightarrow \infty} \int_{0}^{\infty} \mathbb{N}(x) F(t x) d x=0$.

Proof:

Suppose that

$$
f(x)= \begin{cases}c & x \in[\alpha, \beta] \subset[0, \infty) \\ 0 & x \in[0, \infty) \backslash[\alpha, \beta],\end{cases}
$$

then we have: 
$(3.63)$

$$
\begin{aligned}
& \int_{0}^{\infty} f(x) F(t x) d x=c \int_{\alpha}^{\beta} F(t x) d x \\
& =\frac{c}{t} \int_{0}^{\beta t} F(s) d s-\frac{c}{t} \int_{0}^{\alpha t} F(s) d s,
\end{aligned}
$$

so we conclude that for this particular choice of $f(x)$

$$
\lim _{t \rightarrow \infty} \int_{0}^{\infty} f(x) F(t x) d x=0 .
$$

Now $f(x) \in L,[0, \infty)$, so for any $\varepsilon>0$ there exists a simple function $g_{m}(x)=\sum_{i=1}^{m} f_{i}(x)$, such that

$$
\int_{0}^{\infty}\left|f(x)-g_{m}(x)\right| d x<\varepsilon,
$$

where the $f_{i}(x)$ are of the form

$$
f_{i}(x)= \begin{cases}c_{i} & x \in\left[\alpha_{i}, \beta_{i}\right] \subset[0, \infty) \\ 0 & x \in[0, \infty) \backslash \backslash\left[\alpha_{i}, \beta_{i}\right], .\end{cases}
$$

and the $\left[\alpha_{i}, \beta_{i}\right]$ are patrwise disjolnt $[12]$ p. 67. Then the following inequality becomes obvious:

$$
\begin{aligned}
\left|\int_{0}^{\infty}-f(x) F(t x) d x\right| & \leq\left|\int_{0}^{\infty}\left\{f(x)-g_{m}(x)\right\} F(t x) d x\right| \\
& +\left|\int_{0}^{\infty} F(t x) g_{m}(x) d x\right| \\
& =I_{1}+I_{2} .
\end{aligned}
$$

Now $F(x)$ is bounded, $|F(x)| \leqslant M$, and

$$
I_{1} \leqslant M \int_{0}^{\infty}\left|f(x)-g_{m}(x)\right| d x<\varepsilon M .
$$


From the definition of $g_{m}(x)$ we deduce that

$$
\int_{0}^{\infty} g_{m}(x) F(t x) \cdot d x=\sum_{i=f}^{m} \int_{0}^{\infty} f_{i}(x) F(t x) \cdot d x
$$

and since

$$
\lim _{t \rightarrow \infty} \int_{0}^{\infty^{-}} f_{i}(x) F(t x) d x=0 \text { for } 1=1, \ldots, m, .
$$

we conclude that

$$
\lim _{t \rightarrow \infty} \int_{0}^{\infty} g_{m}(x) F(t x) d x=0 .
$$

Now there exists a $\mathrm{T}>0$ such that $\forall \mathrm{t}>\mathrm{T}, \mathrm{I}_{2}<\varepsilon$. Hence for $t>T$,

$$
\left|\int_{0}^{\infty} f(x) F(t x) d x\right|<\varepsilon(M+1), \quad \text { i.e. }
$$

$$
\lim _{t \rightarrow \infty} \int_{0}^{\infty} f(x) F(t x) d x=0 .
$$

It. can be shown similarly that

$$
\lim _{t \rightarrow-\infty} \int_{0}^{\infty} f(x) F(t x) d x=0
$$

Hence

$$
\begin{aligned}
& \begin{array}{l}
\lim \\
|t| \rightarrow \infty
\end{array} \int_{0}^{\infty} f(x) F(t x) d x=0 . \\
& \text { As a result of this Lemma we conclude that } \\
& \lim _{|t| \rightarrow \infty} \int_{0}^{\infty} f(x) \cos t x d x=0, \text { and } \\
& \lim _{t \rightarrow \infty} \int_{0}^{\infty} f(x) \sin t x d x=0,
\end{aligned}
$$

for all $f \in L,[0, \infty)$. 
43

Lemma 8

For large values of $\lambda$ we have

$$
\begin{aligned}
& A(0, \lambda) \sim \exp \{-\lambda \mathrm{h}\} / \lambda, \\
& A_{0}(\lambda) \sim \exp \left\{+\lambda_{\mathrm{h}}\right\} / \lambda, \\
& \mathrm{B}_{0}\left(\lambda^{\prime}\right) \sim \exp \left\{-\lambda_{\mathrm{h}}\right\} / \lambda, \\
& \mathrm{C}_{0}(\lambda) \sim \exp \left\{-\lambda_{\mathrm{h}}\right\} / \lambda .
\end{aligned}
$$

Proof :

We have shown in Lemma 5 that, for large values of (3.47) (a) and (b) has a solution for ail $x$, in particular $\phi(0, \lambda)$ exists. Also from $(3.62)$ we have: (3.64) $\quad \phi(0, \lambda)=\lambda\left[-1+0\left(1 / \lambda^{2}\right)\right]$.

From (3.58) we obtain by differentiation (3.65) $\quad A^{\prime}(0, \lambda)-\phi(0, \lambda) A(0, \lambda)=0$, for all $\lambda \geqslant \lambda$.

From $(3.4)(a)$ - (d) and $(3.65)$ we have for ali values of $\lambda \geqslant \lambda_{0}$ :

$$
(3.66)(a) \quad A(0, \lambda)-\frac{\mu_{0}(\dot{I} / \pi) \exp \{-\lambda \mathbf{h}\}}{\lambda-\left(\mu_{0} / \mu(0)\right) \emptyset(0, \lambda)} \text {. }
$$

(b) $\quad A_{0}(\lambda)=A(0, \lambda)+\mu_{0} \frac{I}{\pi} \frac{\exp \{-\lambda h\}}{2 \lambda}[\exp \{2 \lambda h\}-1]$

(c) $B_{0}(\lambda)=A(0, \lambda)-\mu_{0} \frac{I}{\pi} \frac{\exp \{-\lambda h\}}{2 \lambda}$

(d) $\quad c_{0}(\lambda)=\mu_{0} \frac{I}{\pi} \frac{\exp \{-\lambda \mathrm{h}\}}{2 \lambda}$.

If we substitute $(3.64)$ into $(3.66)(a)$ wo obtain for large values of $\lambda$ :

$$
{ }^{\prime} A(0, \lambda)=\frac{\mu_{0}(I / \pi) \exp \{-\lambda h\}}{\lambda\left[1+\frac{\mu_{0}}{\mu(0)}+\frac{\mu_{0}}{\mu(0)} 0\left(\frac{1}{\lambda^{2}}\right)\right]} .
$$


Thus we have demonstrated the asymptotic behaviour of $A(0, \lambda), A_{0}(\lambda), B_{0}(\lambda)$, and $C_{0}(\lambda)$ proposed in the Lemma.

(

Lemma 9

$A(0, \lambda), A_{0}(\lambda), B_{0}(\lambda), C_{d}(\lambda)$, and $A(x, \lambda)$ may be determined from $(3.4)(a)-(f)$ for all $0 \leqslant \lambda<\infty$, and for all $0<x<\infty$.

Froof:

From $(3.46)$ and $(3: 41)(b)$ we determine $A(x, \lambda)$ and $A(0, \lambda)$ in terms of $a_{2}(x, \lambda), a_{2}(0, \lambda)$, and $a_{2} \cdot(0, \lambda)$. since $a_{2}(x, \lambda)$, and hence $a_{2}(0, \lambda)$ and $a_{2} \cdot(0, \lambda)$, are assumed to be known, we have

$$
\begin{aligned}
& \text { (3.h7)(a) } \quad A(n, \lambda)-\frac{\mu_{0}\left(\mu(0) \%_{\pi}\right) I \exp \{-\lambda \mathrm{h}\}}{\mu(0) \lambda \mathrm{a}_{2}(0, \lambda)-\mu_{0} a_{2}(0, \lambda)} a_{2}(0, \lambda)
\end{aligned}
$$

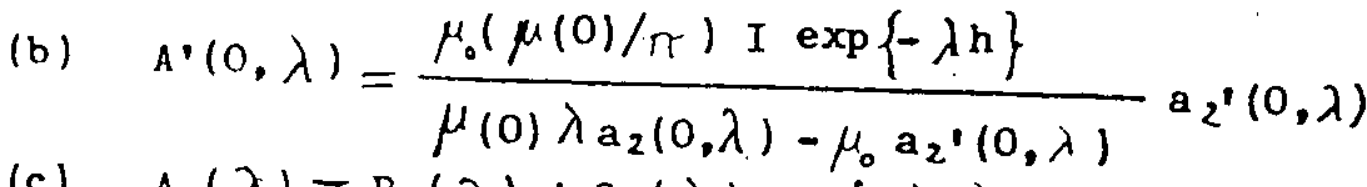

$$
\begin{aligned}
& \text { (c) } A_{0}(\lambda)=B_{0}(\lambda)+c_{0}(\lambda) \exp \{2 \lambda h\} \\
& \text { (d) } B_{0}(\lambda)=A(0, \lambda)-C_{0}(\lambda) \\
& \text { (e) } \quad c_{0}(\lambda)=\frac{I}{2 \pi} \frac{\mu_{0} \exp \{-\lambda h\}}{\lambda} \text {. }
\end{aligned}
$$

3. The Proof of the Theorem

Proof of the Theorem:

With the aid of the nine Lemuas we may now proceed to. prove the theorem. 
Since we have the asymptotic behaviour in $\lambda^{2}$ for * $A_{0}(\lambda), B_{0}(\lambda), C_{0}(\lambda), A(0, \lambda)$, and $A(x, \lambda)$, and sínce all three integrands in $(3.1)-(3.3)$ are continuous, all three integrals converge. Here we have defined the values of the Integrands at $\lambda_{0}=0$ as the value of lim

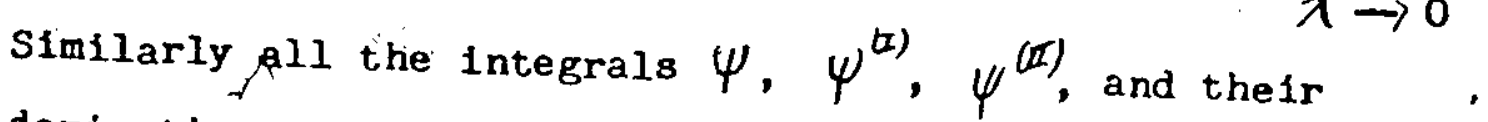
derivatives up to the second order converge. The integrands for $\psi^{(2)}, \psi^{(\pi)}$, and $\psi$ also satisfy the condition at infinity. The latter follows from Lemmas 4 and 7 .

If we substitute. $\psi^{(x)}, \psi^{(\pi)}$, and $\psi$ into the differential equations and the bourdary conditions and 知唯ake use of the Dirichlet Integral

$$
\int_{0}^{\infty} \operatorname{dos} \lambda u \frac{\sin \lambda L}{\lambda L} d \lambda= \begin{cases}\frac{\pi}{2 L} & |u|<L \\ 0 & |u|>L\end{cases}
$$

we see that they satisfy the differential equations and the boundary conditions: Hence the representation theorem. his been proved. 
Chapter 4

\section{APPROXIMATE METHODS}

The representation theorem of the last chapter shows that one of the integrands is composed of the solution of a sécond order linear differential equation. Since it is well known that the exact solution of 8 second order linear differential equation with non-constant coefficients cannot be obtained using a finite number of algebraic, differential, or integral operations $[/ /]$ p. 26 , it becomes imperative to provide some methods for approximating the solution. Because the engineer and physicist are also interested in the quantitative aspects of the solution, only those approximate solutions are discussed which can be evaluated numerically on a computer. For computational reasons, we are interested only in the values of $\psi(x, \mu)$ in the range of $x$ which is of the order of the skin depth being heated., The frequency used in induction hardening is so high that the skin depth is very small compared with the radius of the cylinder, hence it suffices to have approximate methods which are good for small values of $x$ only: We note also that in applications the permeability and conductiolty are functions which vary relatively 
slowly, that is, deviate from a constant value by a function whose order of magnitude is very small compared to that of the constant. We shall make use of this property in constructing the approximate solution.

1. The Layer Method

The Layer Method consists essentially of replacing the model previously described -- where the permeability $\mu(x)$ and the conductivity $\sigma(x)$ are assuned to depend continuously upon the spatial coordinate $x$ - by a model which may be characterized by postulating that the. permeability $\mu_{\nu}$ and the conductivity $\sigma_{\nu}$ in the metallic medium which occupies the half space $R=\{(x, u) \mid x \geqslant 0$, $|u|<\infty\}$ are constants in the layers $L_{\nu}=\{(x, u) \mid$ $(\nu-1) \Delta x \leq x \leq \nu \Delta x,|u|<\infty\} \nu=1, \cdots, \cdots$, , of uniform width $\Delta x$.

If we adopt this layer model, we will obtain the following system of differential equations and boundary conditions :

$$
\begin{gathered}
\Delta \psi^{(\nu)}=0, \\
\Delta \psi^{(\nu)}=0, \\
\Delta \psi^{(\nu)}+k_{\nu}^{2} \psi^{(\nu)}=0 \\
\text { where } k_{\nu}^{2}= \begin{cases}-1 \omega \mu_{\nu} \sigma_{\nu} & \nu=1, \\
0 & \nu=0,\end{cases}
\end{gathered}
$$


subject to:

48

$$
\begin{aligned}
& \frac{\partial \psi^{(I I)}}{\partial x}-\frac{\partial \psi^{(I)}}{\partial x}= \begin{cases}\mu \frac{I}{2 L}, & \mathbf{x}=-\mathbf{h}, \quad|u|<\mathrm{L} \\
0, & \mathbf{x}=-\mathrm{h}, \quad|u|>\mathrm{L}\end{cases} \\
& \frac{\partial \psi^{(\pi)}}{\partial u}-\frac{\partial \psi^{(2)}}{\partial u}=\phi \\
& x=-h, \quad|u|<\infty \\
& \psi^{(x)}-\psi^{(x)}=0, \\
& x=-h, \quad|u|<\infty \\
& \frac{\partial \psi^{(I)}}{\partial u}-\frac{\partial \psi^{(1)}}{\partial u}=0 \text {, } \\
& x=0, \quad|u|<\infty \\
& \mu_{1} \frac{\partial \psi^{(I)}}{\partial x}-\mu_{0} \frac{\partial \psi^{(\prime)}}{\partial x}=0 \text {, } \\
& x=0, \quad|u|<\infty \\
& \psi^{(x)}-\psi^{(0)}=0 \text {, } \\
& \mathbf{x}=0, \quad|u|<\infty \\
& \frac{\partial \psi^{(\nu)}}{\partial u}-\frac{\partial \psi^{(\nu+1)}}{\partial u}=0, \\
& x=\nu \Delta x,|u|<\infty \\
& \mu_{\nu+\cdot} \frac{\partial \psi^{(\nu)}}{\partial x}-\mu_{\nu} \frac{\partial \psi^{(\nu+1)}}{\partial x}=0 \text {, } \\
& \psi^{(j)}-\psi^{(j+1)}=0 \text {, } \\
& \psi^{(n+1)}=\psi_{x}^{(n+1)}=\psi_{u}^{(n+1)}=0 . \\
& x=\nu \Delta x,|u|<\infty \\
& 1, \cdots \cdots N \\
& x=\nu \Delta x,|u|<\infty
\end{aligned}
$$

Wo then use the following representations to approximate the solution of the above boundary value problem:

$$
\begin{aligned}
(4.1): \tilde{\psi}^{\prime}(x, u)= & \int_{0}^{\infty}\left[B_{0}(\lambda) \exp \{\lambda x\}+c_{0}(\lambda) \exp \{-\lambda x\}\right] \\
& \cos \lambda u \frac{\sin \lambda L}{\lambda L} d \lambda,
\end{aligned}
$$

4 
(4.2) $\tilde{\psi}^{(\lambda)}(x, u)=\int_{0}^{\infty} A_{0}(\lambda) \exp \{\lambda x\} \cos \lambda u \frac{\sin \lambda_{L}}{\lambda L} d \lambda$,

(4.3) $\psi^{(\nu)}(x, \dot{u})=\int_{0}^{\infty} A_{\nu}(\lambda) \exp \left\{-\sqrt{\lambda^{2}-k_{\nu}^{2}} x\right\}$

$$
\cos \lambda \mathbf{u} \frac{\sin \lambda \mathrm{L}}{\lambda \mathrm{L}} \mathbf{d} \lambda,
$$

which use the following relations:

$$
\begin{aligned}
& \text { (4.4) (a) } \quad-A_{0}(\lambda) \exp \{-\lambda h\} \lambda+B_{0}(\lambda) \exp \left\{-\lambda_{h}\right\} \lambda \\
& -c_{0}(\lambda) \exp \{\lambda \mathbf{h}\} \lambda=-\mu_{0} \frac{\mathrm{I}}{\mathrm{j} T} \text {, } \\
& \text { (b) } A_{0}(\lambda) \exp \{-\lambda h\}-B_{0}(\lambda) \exp \{-\lambda h\} \text {. } \\
& 0-C_{0}(\lambda) \exp \{\lambda \mathrm{h}\}=0 \text {, } \\
& \text { (c) } B_{0}(\lambda) \mu_{1} \lambda-c_{0}(\lambda) \mu_{1} \lambda+A_{1}(\lambda) \sqrt{\lambda^{2}-k_{?}^{2}} \mu_{0}=0 \text {, } \\
& \text { (d) }-B_{0}(\lambda)-C_{0}(\lambda)+A_{1}(\lambda)=0 \text {, } \\
& \text { (e) } A_{\nu+1}\left(\lambda \sqrt{\lambda^{2}-k_{\nu+1}^{2}} \mu_{\nu} \exp \left\{-\sqrt{\lambda^{2}-k_{\nu+1}^{2}} \cdot \nu \Delta x\right\}\right. \\
& -A_{\nu}(\lambda) \sqrt{\lambda^{2}-k_{\nu+1}^{p}} \mu_{\nu+1} \exp \left\{\sqrt{\lambda^{2}-k_{\nu}^{2}} \nu \Delta x=0\right. \text {. } \\
& \text { (f) } A_{\nu+1}^{\prime}(\lambda) \exp \left\{-\sqrt{\lambda^{2}-k_{j+1}^{2}} \nu \Delta x\right\} \\
& -A_{\nu}(\lambda) \exp \left\{-\sqrt{\lambda^{2}-k_{\nu}{ }^{2}} \nu \Delta x\right\}=\sigma^{\prime}
\end{aligned}
$$

In order that $(4.4)(e)$ and $(4.4)(f)$ may have $a$ nontrivial solution we require that:

$$
\frac{\mu_{\nu+1}}{\mu_{\nu}}-\frac{\sqrt{\lambda^{2}-k_{\nu+1}^{2}}}{\sqrt{\lambda^{2}-k_{\nu}^{2}}}=0 \text {. }
$$

This can be satisfied approximately when the following hold: 
50

(4.6)(a) $\frac{\sigma_{\nu+1}}{\sigma_{\nu}} \approx 1$,

(b) $\frac{\mu_{\nu+1}}{\mu_{\nu}} \approx 1$.

It is clear that $(4.6)^{\prime}(a)$ and (b) can hold only if $\mu(x)$ and $\sigma(x)$ are slowly varying functions of $x$. In general (4.5) is not always satisfied, the forms (4.1) - (4.4) representing the approximate solution only.

From $(4.4)(2)$ we derive

$(4.7) \quad A_{1}(\nu)=\frac{\mu_{0}}{\pi} I \frac{\exp \{-\lambda \mathrm{h}\}}{\lambda+\left(\mu_{0} / \mu_{1}\right) \sqrt{\lambda^{2}-k_{1}^{2}}}$.

similarly from $(4.5)(a)$ and (b) we derive

$(4.8)$

$$
\begin{aligned}
A_{\nu}(\lambda)= & \frac{\mu_{0}}{\pi} I \frac{\exp \{-\lambda \mathrm{h}\}}{\lambda+\left(\mu_{0} / \mu_{1}\right) \sqrt{\lambda^{2}-k_{1}^{2}}} \\
& \exp \left\{-\sum_{j=0}^{\nu-1} \sqrt{\lambda^{2}-k_{j}^{2}} \Delta x+\sqrt{\lambda^{2}-k_{\nu}^{2}}(\nu-1) \Delta x\right\} .
\end{aligned}
$$

Substitution of (4.8) into (4.3) yields:

$$
\text { (4.9) } \begin{aligned}
\widetilde{\psi}^{(\nu)}(x, u)= & \frac{\mu_{0}}{\pi} I \int_{0}^{\infty} \frac{\exp \{-\lambda \mathrm{h}\}}{\lambda+\left(\mu_{0} / \mu_{1}\right) \sqrt{\lambda^{2}-k_{i}^{2}}} \\
& \left.\exp \left\{-\sum_{j=1}^{\nu-1} \sqrt{\lambda^{2}-k_{j}^{2}} \Delta x-\sqrt{\lambda^{2}-k_{j}^{2}}\right\}\right\} \\
& \cos \lambda_{u} \frac{\sin \lambda_{L}}{\lambda L} d \lambda,
\end{aligned}
$$

with $x=(\nu-1) \Delta x-\xi, \therefore \Delta x \geqslant \xi \geqslant 0$. 
$5 I$

For $\Delta x \rightarrow 0,(4.9)$ reduces to:

(4.10) $\quad \widetilde{\psi}(x, . u)=\frac{\mu_{0}}{\pi} I \int_{0}^{\infty} \exp \left\{-\int_{0}^{x} \sqrt{\lambda^{2}-k^{2}(s)} d s\right\}$

$$
\begin{aligned}
& \frac{\exp \{-\lambda \mathrm{h}\}}{\lambda+\left(\mu_{0} / \mu(0)\right) \sqrt{\lambda^{2}-\mathrm{k}^{2}(0)}} \\
& \cos \lambda \mathbf{u} \frac{\sin \lambda \mathrm{L}}{\lambda \mathrm{L}} \cdot \mathrm{d} \lambda,
\end{aligned}
$$

and $(4.6)(a)$ and $(b)$ reduce to $\sigma^{\prime} \approx 0, \mu^{\prime} \approx 0$. Even though (4.10) is an integral over an infinite interval, we may approximate it arbitrarily closely by an integral over a finite interval. To see this we can calculate the following:

$$
\begin{aligned}
& \sqrt{\lambda^{2}-k^{2}(x)}=\frac{1}{\sqrt{2}}\left\{\frac{\omega \mu \sigma(x)+1)\left(\sqrt{\lambda^{2}+\omega^{2} \mu^{2}(x) \sigma^{2}(x)}-\lambda^{2}\right)}{\sqrt{\sqrt{\lambda^{4}+\omega^{2} \mu^{2}(x) \sigma^{2}(x)-\lambda^{2}}}}\right\}, \\
& \exp \left\{-\int_{0}^{x} \sqrt{\lambda^{2}-\mathrm{k}^{2}(s)} \mathrm{ds}\right\}=\exp \left\{-\frac{1}{\sqrt{2}} \int_{0}^{x} \frac{\omega \mu(s) \sigma(s) d s}{\sqrt{\sqrt{\lambda^{4}+\omega^{2} \mu^{2}(s) \sigma^{2}(s)}-\lambda^{2}}}\right\} \\
& \cos \frac{1}{\sqrt{2}} \int_{0}^{x} \sqrt{\sqrt{\lambda^{4}+\omega^{2} \mu^{2}(s) \sigma^{2}(s)}-\lambda^{2}} d s \\
& -1 \exp \left\{-\frac{1}{\sqrt{2}} \int_{0}^{x} \frac{\omega \mu(s) \sigma(s)}{\sqrt{\sqrt{\lambda^{4}+\omega^{2} \mu^{2}(s) \sigma^{2}(s)}-\lambda^{2}}} d s\right\} \\
& \therefore \sin \frac{1}{\sqrt{2}} \int_{0}^{x} \sqrt{\sqrt{\lambda^{2}+\omega \mu^{2}(s) \sigma^{2}(s)}-\lambda^{2}} d s \text {, } \\
& \begin{array}{l}
\lambda+\frac{\mu}{\mu(0)} \\
5
\end{array} \\
& \omega \mu(0) \sigma(0) \\
& +1 \frac{\mu_{0}}{\mu(0)} \frac{1}{\sqrt{2}} \sqrt{\sqrt{\lambda^{4}+\omega^{2} \mu^{2}(0) \sigma^{2}(0)-\lambda^{2}}} .
\end{aligned}
$$


52

We now bet $K_{1}(x, \lambda)=\sqrt{\sqrt{\lambda^{4}+\omega^{2} \mu^{2}(x) \sigma \lambda(x)}-\lambda^{2}}$,

$$
\begin{aligned}
& \eta(x, \lambda)=\frac{\omega_{\mu}(x) \sigma(x)}{K_{j}(x, \lambda)}, \\
& \alpha(0, \lambda)=\lambda+\frac{\mu_{0}}{\mu(0)} \frac{1}{\sqrt{2}} \eta(0, \lambda), \\
& \beta(0, \lambda)=\frac{\mu_{0}}{\mu(0)} \frac{1}{\sqrt{2}} \mathrm{~K}_{1}(0, \lambda) \\
& J_{0}(x, \lambda)=\int_{0}^{x} \mathrm{~K}_{1}(t, \lambda) \mathrm{d} t, \\
& \gamma(x, \lambda)=\cos \frac{1}{\sqrt{2}} \mathrm{~J}_{0}(x, \lambda), \\
& \delta(x, \lambda)=-\sin \frac{1}{\sqrt{2}} J_{0}(x, \lambda), \quad \text { and } \\
& J_{1}(x, \lambda)=\int_{0}^{x} \eta(t, \lambda) d t ;
\end{aligned}
$$

whereupon (4.10) becomes:

$$
\text { (i.j1) } \begin{aligned}
\tilde{\psi}(x, u)= & \frac{\mu_{0}}{\pi} \frac{I}{L} \int_{0}^{\infty} \frac{\sin \lambda L}{\lambda} \cos \lambda_{u} \\
& \quad \exp \{-\lambda h\} \exp \left\{-\frac{1}{\sqrt{2}} J_{1}(x, \lambda)\right\} \\
i \quad & {\left[\frac{\alpha(0, \lambda) \gamma(x, \lambda)+\beta(0, \lambda) \delta(x, \lambda)}{\alpha^{2}(0, \lambda)+\beta^{2}(0, \lambda)}\right] d \lambda }
\end{aligned}
$$




$$
\begin{aligned}
& +1 \frac{\mu_{0}}{\pi} \frac{I}{L} \int_{0}^{\infty} \frac{\sin \lambda L}{\lambda} \cos \lambda u \\
& \quad \exp \{-\lambda h\} \exp \left\{-\frac{I}{\sqrt{2}} J_{1}(x, \lambda)\right\} \\
& {\left[\frac{\alpha(0, \lambda) \delta(x, \lambda)-\beta(0, \lambda) \gamma(x, \lambda)}{\alpha^{2}(0, \lambda)+\beta^{2}(0, \lambda)}\right] d \lambda .}
\end{aligned}
$$

If we assume that $\sigma(0) \geqslant\left(8 \mu(0) /\left(\omega \mu_{0}^{2}\right)\right.$ and denoțe the first and second integrahds of $(4.11)$ by $f_{1}(x, u ; \lambda)$ and $f_{2}(x, u ; \lambda)$, then we find that $f_{1}(x, u ; \lambda)$ and $f_{2}(x, u ; \lambda)$ are both dominated by $\exp \{-\lambda h\}$. Wed efine:

(4.12) $\tilde{\psi}_{3}(x, u)=\frac{\mu_{0}}{\pi} \frac{I}{L} \int_{0}^{s}\left[f_{1}(x, u ; \lambda)+1 f_{2}(x, u ; \lambda)\right] d \lambda$

Then for all $\epsilon>0$, we can approximate $(4.11)$ by (4.12) with

$$
\begin{aligned}
& s_{\varepsilon}=\frac{1}{h} \ln \left\{\frac{\mu_{0}}{\pi} \frac{I}{L} \frac{\sqrt{2}}{\varepsilon^{h}}\right\}, \text { i.e. } \\
& \left|\widetilde{\psi}_{s_{\varepsilon}^{\prime}}(x, u)-\widetilde{\psi}(x, u)\right|<\varepsilon .
\end{aligned}
$$

\section{The Method of Integral Equations}

From Lemma 4 of section 2 of Chapter 3 , we have the exdstence of a solution $a_{2}(x, \lambda)$. . We now wish to construct an approximation to this $a_{2}(x, \lambda)$, using the method of integral equations. 
54

From $(3.41)$ (b) wo have:

(4.13) $\quad a_{2}(x, \lambda)=\exp \left\{-\sqrt{\lambda^{2}-k_{x}^{2}} x\right\}$

$$
+\exp \left\{-\sqrt{\lambda^{2}-\mathrm{K}_{\infty}^{2}} x\right\} \mathrm{d}_{2}(x, \lambda) \text {. }
$$

If we let $u(x, \lambda)=\exp \left\{\sqrt{\lambda^{2}-K_{\infty}^{2}} x\right\} d_{2}\left(x_{;} ; \lambda\right)$, we obtain (4.14) $\quad a_{2}(x, \lambda)=\exp \left\{-\sqrt{\lambda^{2}-K_{\infty}^{2}} x\right\}+u\left(x, \lambda_{1}\right)$, where $U(x, \lambda)$ satisfies the integral equation

(4.15)

$$
\begin{aligned}
U(x, \lambda)= & \frac{1}{2 \sqrt{\lambda^{2}-K_{\infty}^{2}}} \int_{x}^{\infty}\left[\exp \left\{\sqrt{\lambda^{2}-K_{\infty}^{2}}(x-t)\right\}\right. \\
& \left.-\exp \left\{\sqrt{\lambda^{2}-K_{\infty}^{2}}(t-x)\right\}\right]\left[K^{2}(t)-K_{\infty}^{2}\right] \\
& {\left[U(t, \lambda)+\exp \left\{-\sqrt{\lambda^{2}-K_{\infty}^{2}} t\right\}\right] d t . }
\end{aligned}
$$

We again employ successive approximations to solve (4.15) by defining:

$$
U_{0}(x, \lambda)=0
$$

$(4.16)$

$$
\begin{aligned}
U_{n}(x, \lambda)= & \frac{1}{2 \sqrt{\lambda^{2}-K_{\infty}^{2}}} \int_{x}^{\infty}\left[\exp \left\{\sqrt{\lambda^{2}-K_{\infty}^{2}}(x-t)\right\}\right. \\
& \left.-\exp \left\{\sqrt{\lambda^{2}-K_{\infty}^{2}}(t-x)\right\}\right]\left(K^{2}(t)-K_{\infty}^{2}\right) \\
& {\left[U_{n-1}(t, \lambda)+\exp \left\{-\sqrt{\lambda^{2}-K_{\infty}^{2}} t\right\}\right] d t . }
\end{aligned}
$$

- The same arguments as ware used in the proof of Lemma 2 permit us to show that:

(4.17)

$$
\begin{aligned}
\left|U_{n}(x, \lambda)-U_{n-1}(x, \lambda)\right| \leqslant \exp \left\{-\operatorname{Real} \sqrt{\lambda^{2}-K_{\infty}^{2}} x\right\} \\
\left.\left.\left.\qquad \frac{\int_{0}^{\infty} \mid K^{2}(t)-K_{\infty}^{2} / d t}{\operatorname{Real} \sqrt{\lambda^{2}-K_{\infty}^{2}}}\right]\right]_{n l}^{n}\right]
\end{aligned}
$$


55

and :

(4.18) $\quad U(x, \lambda)=\sum_{j=0}^{\infty}\left\{U_{j+1}(x, \lambda)-U_{j}(x, \lambda)\right\}$,

that is, the sequence $\left\{U_{n}(x, \lambda)\right\}$ of iterates converges uniformly to a limit function $U(x, \lambda)$. From (4.17) and (4.18) we can derive the following estimate:

(4.19) $\left|\mathrm{U}(x, \lambda)-\mathrm{U}_{n}(x, \lambda)\right| \leqslant \exp \left\{-\right.$ Real $\left.\sqrt{\lambda^{2}-\mathrm{K}_{a}^{2}} x\right\}$

$$
\begin{aligned}
& \sum_{0}^{\infty}\left[\frac{\int_{x}^{\infty} \cdot\left|K^{2}(t)-K_{\infty}^{2}\right| d t}{\operatorname{Real} \sqrt{\lambda^{2}-K_{\omega}^{2}}}\right]^{j} / j i \\
& \left.\leq \sum_{j=\eta}^{\infty}\left[\frac{\int_{x}^{\infty}\left|\mathrm{K}^{2}(\mathrm{t})^{2}-\mathrm{K}_{\omega}^{2}\right| \mathrm{dt}}{\operatorname{Real} \sqrt{\lambda^{2}-\mathrm{K}_{\infty}^{2}}}\right]\right]^{j} / \mathrm{Jl} \\
& \leq M \exp \left\{\frac{\int_{\xi}^{\infty}\left|K^{2}(t)-K_{a}^{2}\right| d t}{\operatorname{ReaI} \sqrt{\lambda^{2}-K_{\infty}^{2}}}\right\} \\
& {\left[\frac{\int_{x}^{\infty}\left|K^{2}(t)-K_{\infty}^{2}\right| d t}{\operatorname{Real} \sqrt{\lambda^{2}-K_{\infty}^{2}}}\right]^{n+1} /(n+1)^{\prime}} \\
& \leq \mu \exp \left\{\frac{\int_{0}^{\infty}\left|K^{2}(t)-K_{\infty}^{2}\right| d t}{\operatorname{Real} \sqrt{\lambda^{2}-K_{\infty}^{2}}}\right\} \text {. } \\
& \therefore \quad \text { where } x<\xi<\infty \\
& {\left[\frac{\int_{0}^{\infty} \cdot\left|K^{2}(t)-K_{\infty}^{2}\right| d t}{\operatorname{Real} \sqrt{\lambda^{2}-K_{\infty}^{2}}}\right]^{n+1} / n+1}
\end{aligned}
$$


From (3.39) we have that

$$
\frac{1}{\operatorname{Real} \sqrt{\lambda^{2}-\mathrm{K}_{\infty}^{2}}} \leq \sqrt{\frac{2}{\omega \mu_{\infty} \sigma_{\infty}}} \quad \text { for all } \lambda \geqslant 0 \text {. }
$$

Hence we have from $(4,29)$

$$
\text { (4.20) }\left|U(x, \lambda)-U_{n}(x, \lambda)\right| \leq y \exp \sqrt{\frac{2}{\omega \mu_{\infty} \sigma_{\infty}}}
$$

$$
\begin{aligned}
& \left.-\int_{0}^{\infty}\left|K^{2}(t)-K_{\omega}^{2}\right| d t\right\} \\
& {\left[\sqrt{\frac{\omega}{2 \mu_{\infty} \sigma}} \int_{0}^{\infty}\left|K^{2}(t)-K_{\infty}^{2}\right| d t\right] /(n+1) !}
\end{aligned}
$$

We now define $a^{(n)}(x, \lambda)=\exp \left\{-\sqrt{\lambda^{2}-k_{i n}^{2}} x\right\}+U_{n}(x, \lambda)$

so that from $(4.14)$ we have:

$$
\begin{aligned}
& \left|a_{2}(x, \lambda)-a^{(n)}(x, \lambda)\right|=\left|u(x, \lambda)-u_{n}(x, \lambda)\right| \\
& \therefore \quad \mu \exp \left\{\sqrt{\frac{2}{\omega \mu_{\infty} \sigma_{\infty}}}\right. \\
& \left.\int_{0}^{\infty}\left|K^{2}(t)-K_{\infty}^{2}\right| d t\right\} \\
& {\left[\sqrt{\frac{2}{\omega \mu_{\infty} \sigma_{\infty}}} \int_{0}^{\infty}\left|K^{2}(t)-K_{\infty}^{2}\right| d t\right]^{n+1} /(n+1) t}
\end{aligned}
$$

We note that the right side of $(4.21)$ may be made arbitrarily small independently of $x$ and $\lambda$ if we choose $n$ sufficiently large. 
3. The Perturbation Method

Using this.method we approach the problem of constructing approximations to $(3.34)$ subject to:

$(4.22)(a) \quad a(0, \lambda)=\frac{\mu_{0}(I / \pi) \exp \{-\lambda h\}}{\lambda+\left(\mu_{0} / \mu(0)\right) \sqrt{\lambda^{2}-K^{2}(\delta)}}$,

and

(4.22)(b) $\quad a^{\prime}(0, \lambda)=-\sqrt{\lambda^{2}-k^{2}(0)}$ a $(0, \lambda)$,

by assuming that $a(x, \lambda)$ can be represented as:

(4.23)(a) $\quad a(x, \lambda)=a_{0}(x, \lambda)+\eta(x, \lambda)$

subject to

(4.23)(b) $\quad \varepsilon(x) \eta(x, \lambda) \simeq 0$;

where $\varepsilon(x)$ is defined by $K^{2}(x)=K^{2}(0)+\varepsilon(x)$ and $a_{0}(x, \lambda)$ is a solution of (4.24) $a_{0}{ }^{n}(x, \lambda)+\left[k^{2}(0)-\lambda^{2}\right] a_{0}(x, \lambda)=0$

subject to the initial conditions $(4.22)(a)$ and $(b)$. Substitution of $(4.23)$ (a) into $(3.34)$ and $(4.22)(a)$ yields :

(b)

$$
\begin{gathered}
\eta^{\prime \prime}(x, \lambda)+x_{0}^{\prime \prime}(x, \lambda)+\left[k^{2}(0)-\lambda^{2}+\varepsilon(x)\right] \\
{\left[a_{0}(x, \lambda)+\eta(x, \lambda)\right]=0}
\end{gathered}
$$

Using the assumptions (4.23)(b) we obtain

$$
\eta^{n}(x, \lambda)+\left[k^{2}(0)-\lambda^{2}\right] \eta(x, \lambda) \cong-\varepsilon(x) a_{0}(x, \lambda) \text {. }
$$


This suggests use of the approximations $\eta(x, \lambda) \cong \tilde{\eta}(x, \lambda)$ where $\tilde{\eta}(x, \lambda)$ is defined by (4.26) $\tilde{\eta}^{n}(x, \lambda)+\left[k^{2}(0)-\lambda^{2}\right] \tilde{\eta}(x, \lambda)=-\varepsilon(x) a_{0}(x, \lambda)$. subject to $(4.25)(b)$. The solution is found to be

$$
\begin{aligned}
& \text { (4.2.7) } \tilde{\eta}(x, \lambda)=a_{0}(0, \lambda) \frac{\int_{0}^{x}-\varepsilon(s) d s}{\sqrt[2]{\lambda^{2}-K^{2}(s)}} \\
& \exp \left\{-\sqrt{\lambda-K^{2}(0)} \dot{x}\right\} \\
& -a_{0}(0, \lambda) \frac{\int_{0}^{x}-\left\{(s) \exp \left\{-2 \sqrt{\lambda^{2}-k^{2}(0)} \mathbf{s}\right\} \mathbf{d s}\right.}{\sqrt[2]{\lambda^{2}-k^{2}(0)}} . \\
& \exp \left\{\sqrt{\lambda^{2}-k^{2}(0)} x\right\} \text {. }
\end{aligned}
$$

Replacing $\eta(x, \lambda)$ by $\tilde{\eta}(x, \lambda)$ in $(4.23)$ (b) yields the proposed approximation to the equation, which is $(3.34)$ subiect to $(4.22)(a)$ and $(b)$.

4. Some Error Estimates.

From (3.3) we note that the solution $\psi(x ; u)$ is represented by an improper integral with the integrand being the solution of a second order ordinary differential equation subject to two-point boundary conditions. 'In practice, we can not obtain the exact solution of the differential equation using a finite number of steps, while for computational purposes we usually replace the improper 
integral by an integral taken over a finite'interval. In this section we give some error bounds based upon the ábove considerations.

(a) The Error due to Changing the Limits of Integration We have seen that:

(3.3) $\psi(x, u)=\int_{0}^{\infty} A(x, \lambda) \cos \lambda u \frac{\sin \lambda I}{\lambda_{I}} d \lambda$.

If we now deftne:

b:

$(4.28)$

$$
\psi_{\theta}(x, u)=\int_{0}^{\theta} A(x, \lambda) \cos \lambda u \frac{\sin \lambda_{L}}{\lambda_{I}} d \lambda .
$$

we obtain

(4.29) $\quad|\psi(x, u)-\psi(x, u)|<\int_{\theta}^{\infty} \mid A(x, \lambda) \cos \lambda u$

We have en $\frac{\sin \lambda_{L}}{\lambda L} \mid d \lambda$.

We have already shown in Lemma 6 and in Lemma 8 that $|A(x, \lambda)|$ is dominated by $\exp \{-\lambda h\}$ for large values of $\lambda$. We may assume without loss of generality that $\theta$ is sufficiently large that this domination holds and we then" have

$$
\begin{aligned}
& \text { (4.30) } \quad\left|\psi(x, u)-\psi_{\theta}(x, u)\right| \leq \int_{\theta}^{\infty} \exp \{-\lambda \mathrm{h}\} d \lambda \\
& \text { providing that } \\
& =\frac{\exp \{-h \theta\}}{h} \leqslant \varepsilon_{1} \text { ? } \\
& (4.31), \theta=\frac{1}{h} \ln \frac{1}{\mathrm{~h} \dot{\varepsilon}_{l}}
\end{aligned}
$$


tor

60

(b) The Error due to Approximating the Integrand

If we replace $A(x, \lambda)$ by an approximating function $\vec{A}(x, \lambda)$, where $\vec{A}(x, \lambda)$ satisfies:

$$
\begin{aligned}
& \therefore(4.32), \quad|A(x, \lambda)-\bar{A}(x, \lambda)|<\epsilon_{2} \text {. } \\
& \text { and if we define }
\end{aligned}
$$

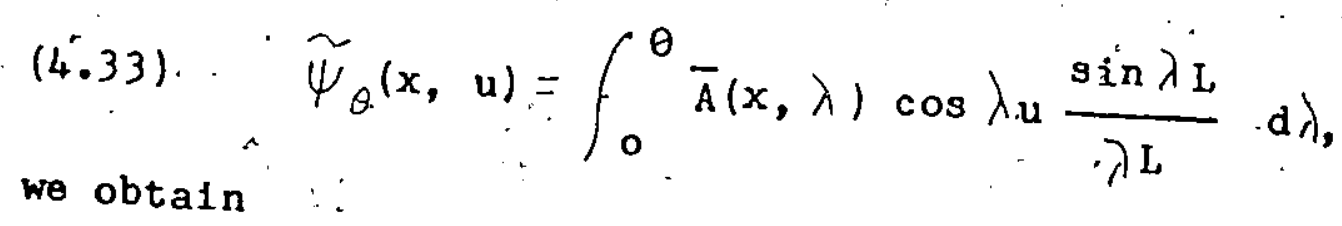

$$
\begin{aligned}
& (4.34) \\
& \left|\dot{\psi}_{\theta}(x, u)-\tilde{\psi}_{\theta}(x, u)\right| \\
& =\left|-\int_{0}^{\theta} A(x ; \lambda)-\bar{A}(x, \lambda) \cos \lambda u \frac{\sin \lambda L}{\lambda_{L}} \ldots d \lambda\right| \\
& \leq \int_{0}^{1}|A(x, \lambda)-\vec{A}(x, \lambda)|\left|\cos \lambda u \frac{\sin \lambda L}{\lambda L}\right| d \lambda \\
& +\int_{I}^{\theta}|A(x, \lambda)-\vec{A}(x, \lambda)|\left|\cos \lambda u \frac{\sin \lambda L}{\lambda L}\right| d \lambda
\end{aligned}
$$

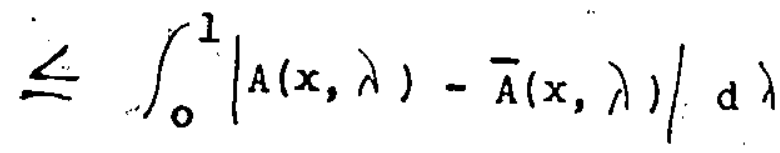

$$
\begin{aligned}
& +\int_{1}^{\theta}|A(x, \lambda)-\vec{A}(x, \lambda)| \frac{1}{L} \frac{d \lambda}{\lambda} \\
& -\varepsilon_{-1}\left[1+\frac{1}{L} \ln \theta\right]
\end{aligned}
$$


By: combining $(4.30)$ and $(4.34)$ we finally obtain

$$
\text { (4.35) } \begin{aligned}
\left|\psi(x, u)-\widetilde{\psi}_{\theta}(x, u)\right| & \leq\left|\psi(x, u)-\psi_{\theta}(x, u)\right| \\
& +\left|\psi_{\theta}(x, u)-\tilde{\psi}_{\theta}(x, u)\right| \\
\leq \varepsilon_{1} & +\varepsilon_{2}\left[1+\frac{1}{L} \ln \theta\right]
\end{aligned}
$$

This error estimate is composed of two parts;

the first due to the use of a finite interval of integration and which may be made arbitrarily small; the second due to approximating the integrand. The latter obviously depends on the goodness of the approximation. 
CHAPTER 5

CONCLUSTON

This study has been based upon a physical problem of considerable interest to industry and technology -that of surface hardening of metallic components to improve their performance characteristics in various mechanisms. The particular application chosen is the surface hardening of a. long, circular rod by the use of induction heating, for which the mathematical formulation of the electromagnetic part of the problem has been chosen for study.

The case of the cylindrical rod has been formulated mathematically as a system of differential equations with coupled boundary conditions. A simplefication in this proble has been made by approximating it by a problem in the half plane. The representation theorem for the solution of the latter problem is then stated and proved. Being an applied problem, some methods for evaluating the solution have been provided. These are the layer method, the method of integral equations, and the perturbation method, together with some error estimates. It is hoped that this presentation will be useful not only for the present problem, but also as a method for attacking other problems in engineering and physics having similar formulations: 
Few investigators are so blessed as to work on a problem to which they can supply all the solutions, and this problem likewise leaves questions unanswered. Some further topics which require investigation in order to further facilitate the more general adoption of the method of surface hardening by induction heating will be mentioned in closing.

The first physical problem that coines to mind is that of the end conditions, that is to say, the effects on the rod on entering and leaving the coil. The consideration of this problem will involve much more complicated statements of boundary conditions, but will be of condiderable interest in applications. Another physical problem concerns the hardening of workpieces of different shapes. At the outset it is obvious that the simplification to a plane problem is not likely possible for even sirple shapes to be used. The addition of another dimension as well as the new boundary conditions will be the principle difficulties met in this kind of extension.

From the fact that induction hardening is reproducible in practice, we infer that the solution is unique. Another mathematical problem for investigation is that of showing that the solution for any of the problems formulated has a solution which is unique. 
- The final problem, and that of the greatest importance for engineering applications, is the inverse problem. That is to say, the geven workpiece has a specified shape and is to be hardened in a desired pattern, which need not be uniform or even continuous. Many parameters are required to be defined for the set up of the process, some of which may permit alternative combinations which may involve optimizing trade offs in application. The shape of the coil to conform to the shape of the workplece is the most obvious parameterich may be varied. Another involves the end effects, or the orientation' of small workpieces for presentation to the process. Velocity of passage, and the frequency and intensity of the current in the coil are other parameters that come readily to mind as being available for variation.

While the inverse problem is probably beyond the possibility of mathematical analysis in the near future, its approximation empiricaliy or heuriftically is needed for the general adoption of the method in manufacturing technology.

It is hoped that these thoughts may encourage others to consider the further ramifications of the problem. 
REFERENCES
[1]. Beckenbach, E. F. and Bellman, $R$. (1970)
$\left[2^{-}\right]$Beilghan, $R$.
[3] Birkhoff, G. D. (1908)

$[4] \underset{(1934)}{\text { Buchholz }}, \mathrm{H}$.

[5] Buchholz, H.

[6] $\underset{(1957)}{\text { Buchholz }}, \stackrel{H}{\text {. }}$

$[7] \underset{(1971)}{\text { Cesari, L. }}$

$[8] \underset{\substack{\text { Jackson, } \\(1962)}}{\mathrm{J}}$
Inequalities,

Sec ond Revised Printing, Springer-Verlag, New York. Methods of Nonlinear Analysis,
Academic Press, Now York.

On the Asymptotic Character of the Solution of Certain Linear Differential Equations Containing a Parameter, Transactions of the American Mathematical Society, Vol. 9, (219-231).

Die Wechselstromgespeiste spule endlicher wicklungshọe mit unendlich langen metallischen Kern, Wissenschaftliche ritteilung der $A E G$, Band XXVIII.

Elektrische und magnetische Potentialfelder, Berlin, Springer Seite $309,6.32$ ia.

Das Magnetfeld-der Wirbelstrome in einem elektrischen Induktions-


Wirbelstromfsider, Arohiv fur Elëktrotechnik,
43 Band, 6 Heft.

A Symptotic 'Behaviour and Stability Problems in Ordinary Differential Equations, Third Edition, Springer-Verlag, New York. Classical Electrodynamics, John Wlley and Son, New York,
page 225 . 




$[14] \underset{(1963)}{\text { Zischka }}, \mathrm{K} . \mathrm{A}$.

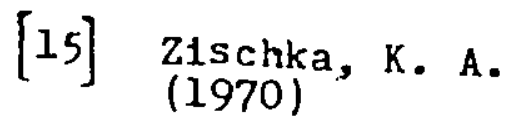

The Asymptotic Solution of Certain Linear Ordinary Differenti Equations of Second Order, Transactions of the American diathematical Society, Vol. 36 (90-106).

Industrial Applications of Induction Heating, Pergamon Press, New York. Ordinary Differential Equations, Prent1ce-Hall, Englewood Cliffs, page 26.

Real and Complex Analysis; McGraw-Hill, New York,
page 67.

Mathematical Hiodels in the Stoel Industry, Proceedings of the conference on Mathematical Models in Metallurgical Process Development organized by the Iron and Steel Institute, The Iron and Steel Institute Publication, 123 , page 2 .

Beitrag zur Mathematischen Theorle der Induktionshartung, Dr. rer. nat. Dissertation der Technischen Hochschule, Darmstadt.

Beltrag zur Niathematischen Theorie der Induktionshartung, Teil I, Elektrischer Teil, Sonderdruck aus 'Elektrowärme International', Band 28, Nr. 3. 
VITA AUCTORIS

67

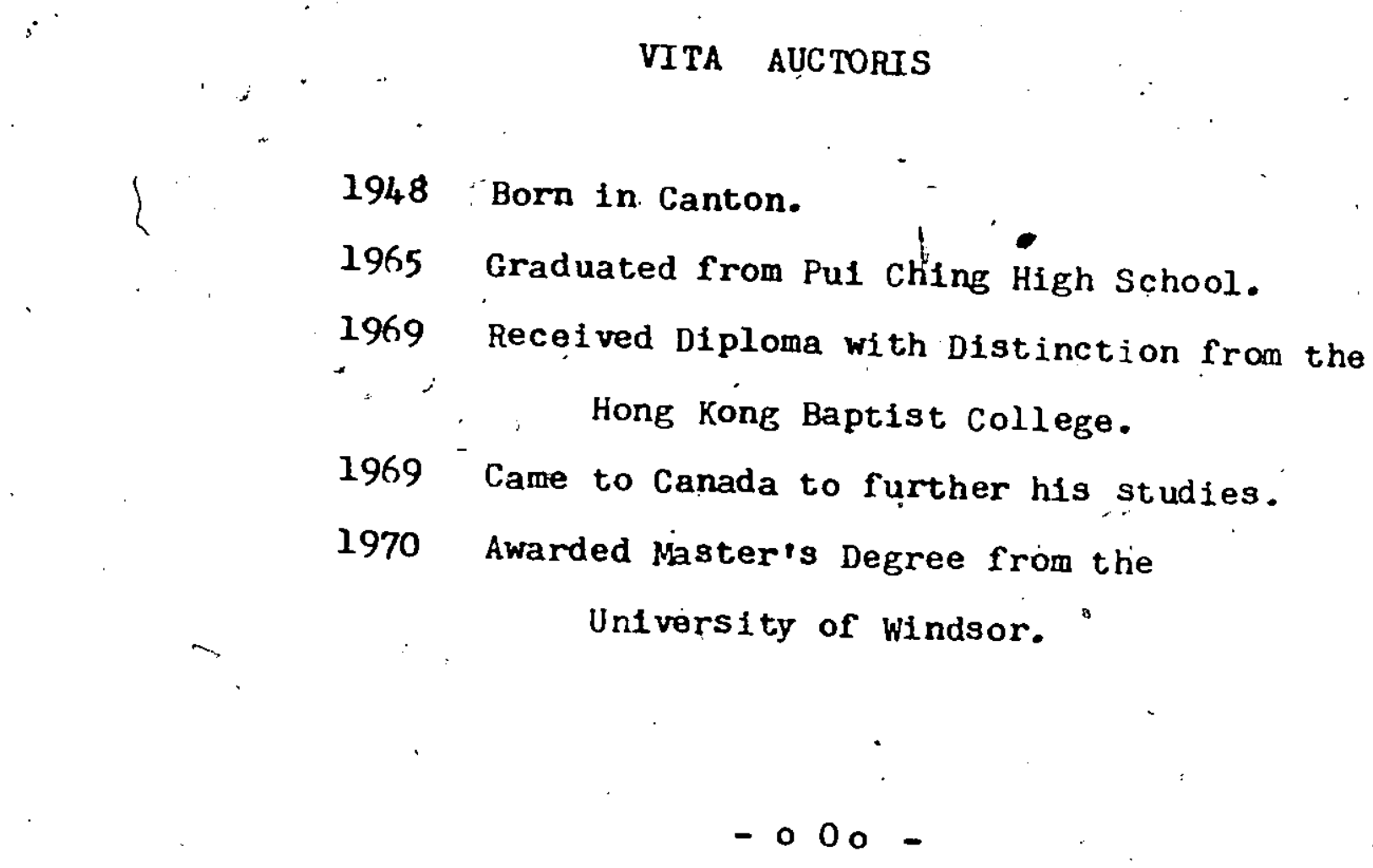

